\title{
Meromorphic Symbolic Structures for Boundary Value Problems on Manifolds with Edges
}

\author{
G. De Donno and B.-W. Schulze
}

\begin{abstract}
We investigate the ideal of Green and Mellin operators with asymptotics for a manifold with edge-corner singularities and boundary which belongs to the structure of parametrices of elliptic boundary value problems on a configuration with corners whose base manifolds have edges.
\end{abstract}

AMS Classification 35S15, 35J70, 58J32

\section{Contents}

Introduction 1

1. Mellin and Green operators in the cone calculus 3

2. Symbols with values in the cone algebra 16

3. Kernel cut-off for cone operators where the base is a manifold with edge

References

\section{Introduction}

Parametrices of elliptic boundary value problems for differential operators on a manifold with smooth boundary belong to a pseudo-differential calculus with a symbolic hierarchy (interior and boundary symbols) and with typical contributions from the boundary (Green, trace, and potential operators), cf. Boutet de Monvel [1]. Similar structures may be obtained for the case of manifolds with geometric singularities, e.g., conical points, edges, corners, etc., as they are natural in a number of applications. Such problems belong to the analysis of operators on stratified and non-compact spaces. However, as is known from the analysis for conical and edge-singularities, answers are far from being straightforward. This concerns, in particular, the regularity and asymptotics of solutions near the singularities, the nature of extra conditions along lower-dimensional skeleta (including topological 
abstructions for their existence), and the description of analogues of Green functions (Green operators). Another experience from the known scenario for conical singularities is that meromorphic operator functions operating on the base of corners play a crucial role, both as the conormal symbolic structure and for evaluating the asymptotics of solutions and relative indices under changing of weights. In the case of corner singularities, where the base itself has conical points or edges, this has to be combined with an edge symbolic calculus. These ingredients contribute to Green (plus Mellin) operators. The program of this paper is to characterise the corresponding algebra of Green (plus Mellin) operators for the case of boundary value problems on a manifold with edges and corners.

The geometry near the corner points is that of a local cone where the base is a manifold with edges and boundary (that means, our configuration has edges with conical singularities and in addition a boundary). Similar structures for the case without boundary have been investigated in [10]. Other simpler special cases are manifolds with smooth edges and boundary; this is studied systematically in the monograph of Kapanadze and Schulze [2], motivated by applications in crack theory. In particular, in such models it is interesting to analyse the mechanism of how solutions to elliptic boundary value problems 'acquire' asymptotics close to the singularities. By Kondratyev's work [3] this is a famous story for conical singularities with smooth base manifolds. Later on many other special cases have been studied, cf. the references in [10] or [2].

For corner singularities the asymptotic information is complicated, already from the point of view of discribing the singular functions in functional analytic terms. For 'simple' corners (i.e., when the base is a (closed) manifold with conical singularities) this is done in [5]. The case when the base is a (closed) manifold with edges is treated in [10]. In all these theories it is importent to understand the nature of parameter-dependent operators on manifolds with conical or edge singularities. The dependence on the parameter is degenerate in some way (here 'edge-degenerate') and the contributions from the given operators to asymptotics are governed by a hierarchy of various principal symbolic components. Roughly speaking, there is always a splitting of the operator algebras into a 'flat' and an 'asymptotic' part; they determine together the elliptic regularity in a specific way. Flat operators on manifolds with edge-corner singularities and boundary have been investigated in Oliaro and Schulze [4]. In the present paper we study the complementary algebra of Green plus Mellin operators belonging to the 'full' structure generated by both parts. Along the edges we adopt the concept of continuous asymptotics (in axial direction of the inner model cone) caused by the (in general very complex) behaviour of variable and branching poles of meromorphic operator functions; along the corner axis we will consider discrete asymptotics.

Following the idea of symbolic hierarchies to organise the calculus for higher singularities by repeated 'conification' and 'edgification' of an already achieved structure, we start from conical singularities in simplest form and describe spaces with asymptotics for this case. We then pass to the 'asymptotic' part of the cone algebra by describing the corresponding Green and Mellin operators. Everything 
is done for compact base manifolds with smooth boundary. The next floor of the hierarchy is that we study operator-valued symbols taking values in the algebra of Mellin and Green operators on an infinite cone. We will need a variant with holomorphic dependence on covariables which we obtain by applying a kernel cutoff procedure. We show how the structure of smoothing Green plus Mellin symbols is preserved in this process (this is remarkable for the Mellin part because of a very specific dependence of cut-off functions on the covariables). After a globalisation for a (stretched) manifold $\mathbb{W}$ with edges and boundary we obtain Green and Mellin operators on $\mathbb{W}$ with parameters. They represent the raw material for our final conormal symbolic structure which we construct by applying another kernel cutoff in the covariable to the corner axis variable. In a concluding section we show the shape of edge-corner degenerate operators which are the essential information for the complete algebra to be generated by the present calculus together with the flat operators mentioned before.

\section{Mellin and Green operators in the cone calculus}

\subsection{Asymptotics.}

1.1.1. Weighted Sobolev spaces. Given a topological space $X$ we define the cone $X^{\triangle}:=\left(\overline{\mathbb{R}}_{+} \times X\right) /(\{0\} \times X)$ as the quotient space, with $\{0\} \times X$ being identified with a point, the tip of the cone. Moreover, we set $X^{\wedge}:=\mathbb{R}_{+} \times X$, called an open stretched cone with base $X$. In our case the base $X$ will be either a compact $C^{\infty}$ manifold with boundary, or a closed $C^{\infty}$ manifold. If $X$ is a $C^{\infty}$ manifold with boundary, we also employ $N:=2 X$, the double of $X$, defined by gluing two copies $X_{ \pm}$of $X$ along their common boundary in a canonical way.

To define Green operators of the cone calculus we first give a definition of weighted Sobolev spaces and subspaces with asymptotics on the (infinite stretched) cones $X^{\wedge}$ and $N^{\wedge}$, respectively. In the following considerations we admit $N$ to be an arbitrary closed compact $C^{\infty}$ manifold of dimension $n$.

Let $L_{\mathrm{cl}}^{\mu}\left(N ; \mathbb{R}^{l}\right)$ denote the space of all classical parameter-dependent pseudodifferential operators of order $\mu \in \mathbb{R}$ on $N$. This means that in local (classical) amplitude functions $a(x, \xi, \lambda)$ the parameter $\lambda \in \mathbb{R}^{l}$ is considered as a part of the covariables $(\xi, \lambda)$, and $L^{-\infty}\left(N ; \mathbb{R}^{l}\right)$ is defined as $\mathcal{S}\left(\mathbb{R}^{l}, L^{-\infty}(N)\right.$ ) (the Schwartz space of $L^{-\infty}(N)$-valued functions) with $L^{-\infty}(N) \cong C^{\infty}(N \times N)$ being equipped with its natural Fréchet topology.

Let $H^{s}(N), s \in \mathbb{R}$, denote the scale of standard Sobolev spaces on $N$. It is then well known that $L_{\mathrm{cl}}^{\mu}\left(N ; \mathbb{R}^{l}\right)$ for every real $\mu$ contains an element $R^{\mu}(\lambda)$ which is parameter-dependent elliptic of order $\mu$ and induces isomorphisms

$$
R^{\mu}(\lambda): H^{s}(N) \longrightarrow H^{s-\mu}(N)
$$

for all $s \in \mathbb{R}$ and all $\lambda \in \mathbb{R}^{l}$, cf. [8]. We now introduce weighted spaces $\mathcal{H}^{s, \gamma}(\cdot)$ based on the Mellin transform on $\mathbb{R}_{+} \ni r$. The Mellin transform

$$
M u(z)=\int_{0}^{\infty} r^{z-1} u(r) d r
$$


first given for $u \in C_{0}^{\infty}\left(\mathbb{R}_{+}\right)$with $z \in \mathbb{C}$, will be employed in several variants. In particular, $M$ will be extended to larger function and distribution spaces on $\mathbb{R}_{+}$; then $z$ often varies on a weight line $\Gamma_{\beta}:=\{z \in \mathbb{C}: \operatorname{Re} z=\beta\}$ for some $\beta \in \mathbb{R}$. In addition, we employ $M$ as a transformation on vector-valued functions (where the values belong to a Fréchet space written as a projective limit of Hilbert spaces). Now $\mathcal{H}^{s, \gamma}\left(N^{\wedge}\right)$ for $s, \gamma \in \mathbb{R}$ is defined to be the completion of $C_{0}^{\infty}\left(N^{\wedge}\right) \cong$ $C_{0}^{\infty}\left(\mathbb{R}_{+}, C^{\infty}(N)\right)$ with respect to the norm

$$
\left\{\frac{1}{2 \pi i} \int_{\Gamma_{\frac{n+1}{2}-\gamma}}\left\|R^{s}(\operatorname{Im} z)(M u)(z)\right\|_{L^{2}(N)}^{2} d z\right\}^{\frac{1}{2}} .
$$

Here $R^{s}(\lambda) \in L_{\mathrm{cl}}^{s}\left(N ; \mathbb{R}_{\lambda}\right)$ is an order reducing element in the sense of the relation (1.1), and $n=\operatorname{dim} N$. Details on this type of spaces, may be found in $[\mathbf{6}],[\mathbf{9}]$. In particular, we have $\mathcal{H}^{s, \gamma}\left(N^{\wedge}\right) \subset H_{\text {loc }}^{s}\left(N^{\wedge}\right)$, moreover, $\mathcal{H}^{s, \gamma}\left(N^{\wedge}\right)=r^{\gamma} \mathcal{H}^{0,0}\left(N^{\wedge}\right)$, and $\mathcal{H}^{0,0}\left(N^{\wedge}\right)=r^{-\frac{n}{2}} L^{2}\left(N^{\wedge}\right)$ with $L^{2}$ referring to $d r d x$.

In the sequel it will be convenient to employ the notion of a non-direct sum of Fréchet spaces $E_{0}, E_{1}$ that are embedded in a Hausdorff topological vector space $H$. We then have an isomorphism between $E_{0}+E_{1}=\left\{e_{0}+e_{1}: e_{0} \in E_{0}, e_{1} \in E_{1}\right\}$ and $E_{0} \oplus E_{1} / \Delta$ for $\Delta:=\left\{(e,-e): e \in E_{0} \cap E_{1}\right\}$ that turns $E_{0}+E_{1}$ to a Fréchet space, called the non-direct sum of $E_{0}$ and $E_{1}$. In particular, if $E_{0}$ and $E_{1}$ are Hilbert spaces, also $E_{0}+E_{1}$ has a Hilbert space structure by an identification of $E_{0}+E_{1}$ with the orthogonal complement of $\Delta$ in $E_{0} \oplus E_{1}$.

In addition, if a Fréchet space $E$ is a (say, left) module over an algebra $A$, we denote by $[a] E$ for fixed $a \in A$ the completion of $\{a e: e \in E\}$ in $E$.

Observe that $\mathcal{H}^{s, \gamma}\left(N^{\wedge}\right)$ is a $C_{0}^{\infty}\left(\overline{\mathbb{R}}_{+}\right)$-module. Throughout this paper a cut-off function on $\mathbb{R}_{+}$is any real-valued $\omega \in C_{0}^{\infty}\left(\overline{\mathbb{R}}_{+}\right)$such that $\omega \equiv 1$ in a neighborhood of $r=0$.

For purposes below we now modify the $\mathcal{H}^{s, \gamma}$-spaces to a scale $\mathcal{K}^{s, \gamma}$ by imposing far from $r=0$ the structure of 'usual' Sobolev spaces. For the case $N=S^{n}$ (the unit sphere in $\mathbb{R}^{n+1}$ ) we simply take the space $H^{s}\left(\mathbb{R}^{n+1}\right)$. More precisely, if $\chi: \mathbb{R}^{n+1} \backslash\{0\} \rightarrow \mathbb{R}_{+} \times S^{n}$ is the diffeomorphism which describes polar coordinates, we set

$$
H_{\text {cone }}^{s}\left(\mathbb{R}_{+} \times S^{n}\right):=\left\{\left.u\right|_{\mathbb{R}_{+} \times S^{n}}: u \in H_{\text {loc }}^{s}\left(\mathbb{R} \times S^{n}\right),\{(1-\omega) u\} \circ \chi \in H^{s}\left(\mathbb{R}^{n+1}\right)\right\}
$$

for any cut-off functions $\omega(r)$ (clearly this definition is independent of the choice of $\omega$ ). A straightforward generalisation of this definition to $N^{\wedge}$ (using a covering of $N^{\wedge}$ by conical neighborhoods and invariance of elements in $H_{\text {cone }}^{s}\left(\mathbb{R}_{+} \times S^{n}\right)$ supported in a conical set under diffeomorphisms that are homogeneous of order 1 with respect to homotheties in $r$ ) gives us the spaces $H_{\text {cone }}^{s}\left(N^{\wedge}\right)$, cf. [6]. The spaces $H_{\text {cone }}^{s}\left(N^{\wedge}\right)$ can be endowed with Hilbert space scalar products.

We now define

$$
\mathcal{K}^{s, \gamma}\left(N^{\wedge}\right):=[\omega] \mathcal{H}^{s, \gamma}\left(N^{\wedge}\right)+[1-\omega] H_{\text {cone }}^{s}\left(N^{\wedge}\right)
$$

as a non-direct sum which is independent of the specific of the cut-off function $\omega$. 
In $\mathcal{K}^{0,0}\left(N^{\wedge}\right)=\mathcal{H}^{0,0}\left(N^{\wedge}\right)$ we take the scalar product from $r^{-\frac{n}{2}} L^{2}\left(N^{\wedge}\right)$.

If $X$ is a compact $C^{\infty}$ manifold with boundary we set $H^{s}(X):=\left.H^{s}(2 X)\right|_{\operatorname{int} X}$, and

$$
\mathcal{H}^{s, \gamma}\left(X^{\wedge}\right):=\left.\mathcal{H}^{s, \gamma}\left(2 X^{\wedge}\right)\right|_{\operatorname{int} X^{\wedge}}, \mathcal{K}^{s, \gamma}\left(X^{\wedge}\right):=\left.\mathcal{K}^{s, \gamma}\left(2 X^{\wedge}\right)\right|_{\operatorname{int} X^{\wedge}} .
$$

1.1.2. Discrete asymptotics. We now pass to subspaces with asymptotics of our weighted Sobolev spaces on a cone, first for the discrete case. The base will be either a closed compact $C^{\infty}$ manifold $N$ or a compact $C^{\infty}$ manifold $X$ with boundary. To measure asymptotics we fix a reference weight $\gamma \in \mathbb{R}$ and set $\Theta=$ $(\vartheta, 0]$ for some $-\infty \leq \vartheta<0$, interpreted as a weight interval. Then we define

$$
\mathcal{K}_{\Theta}^{s, \gamma}\left(N^{\wedge}\right):=\lim _{\varepsilon>0} \mathcal{K}^{s, \gamma-\vartheta-\varepsilon}\left(N^{\wedge}\right)
$$

and, similarly,

$$
\mathcal{K}_{\Theta}^{s, \gamma}\left(X^{\wedge}\right):=\lim _{\varepsilon>0} \mathcal{K}^{s, \gamma-\vartheta-\varepsilon}\left(X^{\wedge}\right) .
$$

These spaces will be taken in their natural Fréchet topologies. Discrete asymptotics will have the form

$$
u(r, x) \sim \sum_{j} \sum_{k=0}^{m_{j}} c_{j k}(x) r^{-p_{j}} \log ^{k} r
$$

as $r \rightarrow 0$, for certain coefficients $c_{j k} \in C^{\infty}\left(N^{\wedge}\right)$. Notice that $p \in \mathbb{C}$ and $\operatorname{Re} p<$ $\frac{n+1}{2}-\gamma$ entails $\omega(r) c(x) r^{-p} \log ^{k} r \in \mathcal{K}^{\infty, \gamma}\left(N^{\wedge}\right)$ for every $c \in C^{\infty}\left(N^{\wedge}\right), k \in \mathbb{N}$, where $\omega(r)$ is an arbitrary cut-off function. We shall control the coefficients $c_{j k}$ in (1.3) as elements in finite-dimensional subspaces $L_{j} \subset C^{\infty}(N)$ for $0 \leq k \leq m_{j}$, and then call the sequence

$$
P=\left\{\left(p_{j}, m_{j}, L_{j}\right)\right\}_{0 \leq j \leq l}
$$

for a given $l=l(P) \in \mathbb{N} \cup\{\infty\}$ a discrete asymptotic type.

Given weight data $\boldsymbol{g}:=(\gamma, \Theta)$ for $\Theta=(\vartheta, 0]$ we say that $P$ is associated with $g$ if

$$
\pi_{\mathbb{C}} P:=\left\{p_{j}\right\}_{0 \leq j \leq l} \subset\left\{z: \frac{n+1}{2}+\vartheta-\gamma<\operatorname{Re} z<\frac{n+1}{2}-\gamma\right\}
$$

where $l$ is assumed to be finite for finite $\vartheta$ and $\operatorname{Re} p_{j} \rightarrow-\infty$ as $j \rightarrow \infty$ for infinite $l$ and $\vartheta$. Let $\operatorname{As}\left(N, \boldsymbol{g}^{\bullet}\right)$ denote the set of all discrete asymptotic types associated with $\boldsymbol{g}=(\gamma, \Theta)$. For finite $\Theta$ the space of singular functions

$$
\begin{aligned}
& \mathcal{E}_{P}\left(N^{\wedge}\right):=\left\{\omega(r) \sum_{j} \sum_{k=0}^{m_{j}} c_{j k}(x) r^{-p_{j}} \log ^{k} r: c_{j k} \in L_{j}, p_{j} \in \pi_{\mathbb{C}} P,\right. \\
&\left.0 \leq k \leq m_{j}, 0 \leq j \leq l\right\}
\end{aligned}
$$

(for any fixed choice of a cut-off function $\omega$ ), is a finite-dimensional subspace of $\mathcal{K}^{\infty, \gamma}\left(N^{\wedge}\right)$, and we set

$$
\mathcal{K}_{P}^{s, \gamma}\left(N^{\wedge}\right):=\mathcal{K}_{\Theta}^{s, \gamma}\left(N^{\wedge}\right)+\mathcal{E}_{P}\left(N^{\wedge}\right),
$$


endowed with the corresponding Fréchet topology of the sum (which is direct in this case). For the case of infinite $\Theta$ we have the sequence of spaces $\mathcal{K}_{P_{k}}^{s, \gamma}\left(N^{\wedge}\right), k \in \mathbb{N}$, when we take $P_{k} \in \operatorname{As}\left(N, \boldsymbol{g}_{k}^{\bullet}\right)$ with $\boldsymbol{g}_{k}:=(\gamma,(-(k+1), 0])$ and $P_{k}:=\{(p, m, L) \in$ $\left.P: \frac{n+1}{2}-(k+1)-\gamma<\operatorname{Re} p<\frac{n+1}{2}-\gamma\right\}$. Then $\mathcal{K}_{P_{k+1}}^{s, \gamma}\left(N^{\wedge}\right) \hookrightarrow \mathcal{K}_{P_{k}}^{s, \gamma}\left(N^{\wedge}\right)$ is continuous, and we define

$$
\mathcal{K}_{P}^{s, \gamma}\left(N^{\wedge}\right):=\lim _{k \in \mathbb{N}} \mathcal{K}_{P_{k}}^{s, \gamma}\left(N^{\wedge}\right)
$$

Applying this to $N=2 X$ we obtain analogous spaces $\mathcal{K}_{P}^{s, \gamma}(X)$ by restriction of $\mathcal{K}_{P}^{s, \gamma}(2 X)$ to int $X$; at the same time the spaces of coefficients $L_{j}$ are restricted to int $X$, and we write $\operatorname{As}\left(X, \boldsymbol{g}^{\bullet}\right)$ for the corresponding set of discrete asymptotic types.

1.1.3. Continuous asymptotics. The notion of continuous asymptotics employs the observation that asymptotics in the discrete sense, cf. the preceding section, can be represented by meromorphy in the image under the Mellin transform. Let $U \subseteq \mathbb{C}$ be an open set and $E$ a Fréchet space; then $\mathcal{A}(U, E)$ denotes the space of all holomorphic functions in with values in $E$. If $K:=\left\{p_{0}, \ldots, p_{N}\right\} \subset \mathbb{C}$ lies in $\left\{\operatorname{Re} z: z<\frac{n+1}{2}-\gamma\right\}$, and if $f(z) \in \mathcal{A}\left(\mathbb{C} \backslash K, C^{\infty}(N)\right)$ is meromorphic with poles at $K$ of certain multiplicities $m_{j}+1, j=0, \ldots, l$, for every counter clockwise oriented (say smooth) curve $C$ surrounding $K$

$$
\mathcal{A}(\mathbb{C}) \ni h \rightarrow\left\langle\zeta_{f}, h\right\rangle:=\frac{1}{2 \pi i} \int_{C} f(z) h(z) d z
$$

represents an analytic functional $\zeta$ (with poles in $C^{\infty}\left(N^{\wedge}\right)$ ) carried by $K$. Concerning $C$ we always assume that the winding number with respect to every $z \in K$ is equal to 1 (such a choice of $C$ is always possible). To fix notation, if $K \subset \mathbb{C}$ is any compact set, $E$ a Fréchet space, $\mathcal{A}^{\prime}(K, E)$ denotes the space of $E$-valued analytic functionals, carried by $K$. For $E=\mathbb{C}$ we simply write $\mathcal{A}^{\prime}(K)$; this is a (nuclear) Fréchet space, and we then have $\mathcal{A}^{\prime}(K, E)=\mathcal{A}^{\prime}(K) \widehat{\otimes}_{\pi} E$ (here $\widehat{\otimes}_{\pi}$ denotes the (completed) projective tensor product between the respective spaces). Observe that

$$
\left\langle\zeta_{f}, r^{-z}\right\rangle=\sum_{j} \sum_{k=0}^{m_{j}} c_{j k} r^{-p_{j}} \log ^{k} r
$$

for certain coefficients $c_{j k} \in C^{\infty}(N)$ comming from the Laurent coefficients of $f$. We interpret the set $K$ as the carrier of asymptotics of the element $\omega\left\langle\zeta_{f}, r^{-z}\right\rangle \in$ $\mathcal{K}^{\infty, \gamma}\left(\tilde{X}^{\wedge}\right)$ (for any cut-off function $\omega$ ). Notice that the (weighted) Mellin transform of the latter function is meromorphic with the same poles and multiplicities as $f$.

To define continuous asymptotics we pass to more general carrier sets (compact ones for finite weight intervals $\Theta$ ). Let $W^{I}$ for any $W \subset \mathbb{C}$ defined to be the smallest set containing $W$ together with all points $(1-\lambda) w_{0}+\lambda w_{1}, 0 \leq \lambda \leq 1$, $w_{0}, w_{1} \in W$, such that $\operatorname{Re} w_{0}=\operatorname{Re} w_{1}$. By $\mathcal{V}$ denote the system of all closed subsets $V \subset \mathbb{C}$ such that $V \cap\left\{z: c \leq \operatorname{Re} z \leq c^{\prime}\right\}$ is compact for every $c \leq c^{\prime}$ and $V=V^{I}$. Given $V_{1}, V_{2} \in \mathcal{V}$ we write $V=\left(V_{1} \cup V_{2}\right)^{I}$, If are $\boldsymbol{g}=(\gamma, \Theta)$ weight data, first with finite $\Theta=(\vartheta, 0]$, we now define continuous asymptotic 
types $P$ associated with $\boldsymbol{g}$. To this end we choose a compact set $V \in \mathcal{V}$ such that $V \subset\left\{z: \frac{n+1}{2}-\gamma+\vartheta-\varepsilon \leq \operatorname{Re} z<\frac{n+1}{2}-\gamma\right\}$ and form the space

$$
\mathcal{E}_{V}\left(N^{\wedge}\right):=\left\{\omega(r)\left\langle\zeta, r^{-z}\right\rangle: \zeta \in \mathcal{A}^{\prime}\left(V, C^{\infty}(N)\right)\right\}
$$

for some cut-off function $\omega$. Note that there is an isomorphism $\mathcal{E}_{V}\left(N^{\wedge}\right) \cong \mathcal{A}^{\prime}\left(V, C^{\infty}(N)\right)$ induced by $\zeta \rightarrow \omega(r)\left\langle\zeta, r^{-z}\right\rangle$. The inverse map is given by $u \rightarrow \zeta$, for $u=$ $\omega(r)\left\langle\zeta_{z}, r^{-z}\right\rangle$,

$$
\langle\zeta, h\rangle:=\frac{1}{2 \pi i} \int_{C}\left(M_{\gamma-\frac{n}{2}} u\right)(w) h(w) d w
$$

for any smooth curve $C \subset\left\{z: \operatorname{Re} z<\frac{n+1}{2}-\gamma\right\}$ counter clockwise surrounding the set $V$. We thus have a natural Fréchet space structure in $\mathcal{E}_{V}\left(N^{\wedge}\right)$. From the position of $V$ in $\mathbb{C}$ we also obtain

$$
\mathcal{E}_{V}\left(N^{\wedge}\right) \subset \mathcal{K}^{\infty, \gamma}\left(N^{\wedge}\right) .
$$

Notice that when $V \subset\left\{z: \operatorname{Re} z \leq \frac{n+1}{2}-\gamma+\vartheta\right\}$ we have $\mathcal{E}_{V}\left(N^{\wedge}\right) \subset \mathcal{K}_{\Theta}^{\infty, \gamma}\left(N^{\wedge}\right)$ for $\Theta=(\vartheta, 0]$. Writing $u \sim v$ for $u, v \in \mathcal{E}_{V}\left(N^{\wedge}\right)$ when $u-v \in \mathcal{K}_{\Theta}^{\infty, \gamma}\left(N^{\wedge}\right)$ we obtain an equivalence relation in the space $\mathcal{E}_{V}\left(N^{\wedge}\right)$. Then the quotient space $\mathcal{E}_{V}\left(N^{\wedge}\right) / \sim$ is called a continuous asymptotic type $P$ associated with $\boldsymbol{g}$, and we write $\pi_{\mathbb{C}} P:=V \cap\left\{z: \operatorname{Re} z<\frac{n+1}{2}-\gamma\right\}$. Let $\operatorname{As}(N, \boldsymbol{g})$ denote the set of all such continuous asymptotic types. Similarly to (1.5) for $P$ associated with $\boldsymbol{g}=(\gamma, \Theta)$ in terms of $V$ we set

$$
\mathcal{K}_{P}^{s, \gamma}\left(N^{\wedge}\right):=\mathcal{K}_{\Theta}^{s, \gamma}\left(N^{\wedge}\right)+\mathcal{E}_{V}\left(N^{\wedge}\right)
$$

in the Fréchet topology of the non-direct sum.

Let us extend the definition of spaces with continuous asymptotics to the case $\Theta=(-\infty, 0]$ of arbitrary sets

$$
V \in \mathcal{V}, V \subset\left\{z: \operatorname{Re} z<\frac{n+1}{2}-\gamma\right\} .
$$

We first form $V_{k}:=V \cap\left\{z: \operatorname{Re} z \geq \frac{n+1}{2}-(k+1-\gamma)\right\}$ and then obtain for the weight data $\boldsymbol{g}_{k}:=(\gamma,(-(k+1), 0])$ associated continuous asymptotic types $P_{k} \in \operatorname{As}\left(N, \boldsymbol{g}_{k}\right)$ for every $k \in \mathbb{N}$. It can easily be verified that we have continuous embeddings

for all $k$, and we set

$$
\mathcal{K}_{P_{k+1}}^{s, \gamma}\left(N^{\wedge}\right) \hookrightarrow \mathcal{K}_{P_{k}}^{s, \gamma}\left(N^{\wedge}\right)
$$

$$
\mathcal{K}_{P}^{s, \gamma}\left(N^{\wedge}\right):=\lim _{k \in \mathbb{N}} \mathcal{K}_{P_{k}}^{s, \gamma}\left(N^{\wedge}\right)
$$

in the projective limit topology.

In (1.7) by $P$ we undestand a continuous asymptotic type associated with the weight data $(\gamma,(-\infty, 0])$ and represented by the set $V$. It would suffice to simply write ' $V$ ' as subscript, but we prefer the former notation for unifying the descriptions. In this connection we set $\pi_{\mathbb{C}} P=V$ and denote by $\operatorname{As}(N, \boldsymbol{g})$ the set of continuous asymptotic types $P$ (which is bijectively related to the system of all $V \in \mathcal{V}$ for which $\left.V \subset\left\{z: \operatorname{Re} z<\frac{n+1}{2}-\gamma\right\}\right)$. 
Now, for $N=2 X$ we set

$$
\mathcal{K}_{P}^{s, \gamma}\left(X^{\wedge}\right):=\left\{\left.u\right|_{\left(\mathrm{int} X_{+}\right)^{\wedge}}: u \in \mathcal{K}_{P}^{s, \gamma}\left((2 X)^{\wedge}\right)\right\}
$$

for every $P \in \operatorname{As}(2 X, \boldsymbol{g})$ or $\in \operatorname{As}\left(2 X, g^{\bullet}\right)$. To define the topologies we also consider the subspaces

$$
\mathcal{K}^{s, \gamma}\left(X_{-}^{\wedge}\right)_{0} \text { and } \mathcal{K}_{P}^{s, \gamma}\left(X_{-}^{\wedge}\right)_{0}
$$

of elements of $\mathcal{K}^{s, \gamma}\left((2 X)^{\wedge}\right)$ and $\mathcal{K}_{P}^{s, \gamma}\left((2 X)^{\wedge}\right)$, respectively, which are supported by $X_{-}^{\wedge}$. These are, in fact, closed subspaces in the respective topologies, and there are isomorphisms (first algebraic ones)

$$
\mathcal{K}^{s, \gamma}\left(X^{\wedge}\right) \cong \mathcal{K}^{s, \gamma}\left((2 X)^{\wedge}\right) / \mathcal{K}^{s, \gamma}\left(X_{-}^{\wedge}\right)_{0},
$$

and, similarly,

$$
\mathcal{K}_{P}^{s, \gamma}\left(X^{\wedge}\right) \cong \mathcal{K}_{P}^{s, \gamma}\left((2 X)^{\wedge}\right) / \mathcal{K}_{P}^{s, \gamma}\left(X_{-}^{\wedge}\right)_{0}
$$

which give rise to quotient topologies in the spaces (??) and (1.8), respectively.

The asymptotic types $P$ referring to $X$ in such quotient spaces can also be defined directly by replacing $N$ in the above construction by $X$, in other words, we have spaces $\mathcal{A}^{\prime}\left(V, C^{\infty}(X)\right), \mathcal{E}_{V}\left(X^{\wedge}\right)$, etc. Asymptotic types for a manifold $X$ with boundary are then defined by $\mathcal{E}_{V}\left(X^{\wedge}\right) / \sim$, where the equivalence $\sim$ is to be understood analogously to the case $N$. We then obtain a system $\operatorname{As}(X, \boldsymbol{g})$ of continuous asymptotic types also in this case, both for finite and infinite weight intervals, and the subscript ' $P$ ' on the left hand side of (1.9) denotes an element in $\operatorname{As}(X, \boldsymbol{g})$.

For purposes below we also introduce the spaces

$$
\mathcal{S}_{P}^{\gamma}\left(X^{\wedge}\right):=\left\{\omega u+(1-\omega) v: u \in \mathcal{K}_{P}^{\infty, \gamma}\left(X^{\wedge}\right), v \in \mathcal{S}\left(\overline{\mathbb{R}}_{+}, C^{\infty}(X)\right)\right\}
$$

for any cut-off function $\omega$, and $P \in \operatorname{As}\left(X, \boldsymbol{g}^{\bullet}\right)(\in \operatorname{As}(X, \boldsymbol{g}))$.

Example 1.1. The space $\mathcal{S}_{P}^{\gamma}\left(X^{\wedge}\right)$ is nuclear and Fréchet in the topology of the non-direct sum

$$
\mathcal{S}_{P}^{\gamma}\left(X^{\wedge}\right)=[\omega] \mathcal{K}_{P}^{\infty, \gamma}\left(X^{\wedge}\right)+[1-\omega] \mathcal{S}\left(\overline{\mathbb{R}}_{+}, C^{\infty}(X)\right)
$$

(which is independent of the choice of $\omega$ ).

1.2. Green and Mellin operators in the cone algebra.

1.2.1. Green operators with asymptotics on the cone. We now turn to a class of typical elements of the cone algebra of boundary value problems, so called Green operators.

Let $X$ be a $C^{\infty}$ manifold with boundary, $n=\operatorname{dim} X$. Fix a collar neighborhood $V$ of $\partial X$ and choose Riemannian metrics on $X$ and $\partial X$, respectively, such that $V$ corresponds to $\partial X \times[0,1)$ with the product metric. The measures associated with the Riemannian metrics on $X$ and $\partial X$ are denoted by $d x$ and $d y$, respectively. In $V$ we employ the corresponding splitting of variables $x=(y, t), y \in \partial X, t \in[0,1)$. Let $T$ denote any first order differential operator on $X$ with smooth coefficients up to the boundary $Y$ such that $\left.T\right|_{V}=\frac{\partial}{\partial t}$. 
Definition 1.2. By a Green operators of type 0 on the cone $X^{\wedge}$ with continuous asymptotics and weight data $\boldsymbol{g}:=(\gamma, \delta, \Theta)$ we understand a continuous operator

$$
\begin{aligned}
G: \mathcal{K}^{s, \gamma}\left(X^{\wedge}\right) & \oplus \mathcal{K}^{s^{\prime}, \gamma-\frac{1}{2}}\left((\partial X)^{\wedge}, \mathbb{C}^{j_{-}}\right) \\
& \rightarrow \mathcal{S}_{P}^{\delta}\left(X^{\wedge}\right) \oplus \mathcal{S}_{P^{\prime}}^{\delta-\frac{1}{2}}\left((\partial X)^{\wedge}, \mathbb{C}^{j_{+}}\right)
\end{aligned}
$$

such that the formal adjoint $G^{*}$ induces a continuous operator

$$
\begin{aligned}
G^{*}: \mathcal{K}^{s,-\delta}\left(X^{\wedge}\right) & \oplus \mathcal{K}^{s^{\prime},-\delta+\frac{1}{2}}\left((\partial X)^{\wedge}, \mathbb{C}^{j_{+}}\right) \\
& \rightarrow \mathcal{S}_{Q}^{-\gamma}\left(X^{\wedge}\right) \oplus \mathcal{S}_{Q^{\prime}}^{-\gamma+\frac{1}{2}}\left((\partial X)^{\wedge}, \mathbb{C}^{j-}\right)
\end{aligned}
$$

for suitable $(G$-dependent) pairs of asymptotic types

$$
\begin{gathered}
P \in \operatorname{As}(X,(\delta, \Theta)), P^{\prime} \in \operatorname{As}\left(\partial X,\left(\delta-\frac{1}{2}, \Theta\right)\right), \\
Q \in \operatorname{As}(X,(-\gamma, \Theta)), Q^{\prime} \in \operatorname{As}\left(\partial X,\left(-\gamma-\frac{1}{2}, \Theta\right)\right),
\end{gathered}
$$

where the continuity of (1.11) and (1.12) is required for all $s, s^{\prime} \in \mathbb{R}, s>-\frac{1}{2}$ (with $P, P^{\prime}$ and $Q, Q^{\prime}$ being independent of $\left.s, s^{\prime}\right)$ and $G^{*}$ is taken in the sense

$$
\left(u, G^{*} v\right)_{\mathcal{K}^{0,0}\left(X^{\wedge}\right) \oplus \mathcal{K}^{0,-\frac{1}{2}}\left((\partial X)^{\wedge}, \mathbb{C}^{j-}\right)}=(G u, v)_{\mathcal{K}^{0,0}\left(X^{\wedge}\right) \oplus \mathcal{K}^{0,-\frac{1}{2}}\left((\partial X)^{\wedge}, \mathbb{C}^{j+}\right)},
$$

for all $u \in C_{0}^{\infty}\left(X^{\wedge}\right) \oplus C_{0}^{\infty}\left((\partial X)^{\wedge}, \mathbb{C}^{j_{-}}\right), v \in C_{0}^{\infty}\left(X^{\wedge}\right) \oplus C_{0}^{\infty}\left((\partial X)^{\wedge}, \mathbb{C}^{j_{+}}\right)$.

If we require operators $G$ such that (1.11) and (1.12) hold with discrete asymptotic types

$$
\begin{gathered}
P \in \operatorname{As}\left(X,(\delta, \Theta)^{\bullet}\right), P^{\prime} \in \operatorname{As}\left(\partial X,\left(\delta-\frac{1}{2}, \Theta\right)^{\bullet}\right), \\
Q \in \operatorname{As}\left(X,(-\gamma, \Theta)^{\bullet}\right), Q^{\prime} \in \operatorname{As}\left(\partial X,\left(-\gamma-\frac{1}{2}, \Theta\right)^{\bullet}\right),
\end{gathered}
$$

we talk about Green operators with discrete asymptotics. Let $\mathcal{C}_{G}^{0}\left(X^{\wedge}, \boldsymbol{g}\right)$ denote the space of all $G$ as in Definition 1.2, and define $\mathcal{C}_{G}^{d}\left(X^{\wedge}, \boldsymbol{g}\right)$ for $d \in \mathbb{N}$ as the space of all operators of the form

$$
G=G_{0}+\sum_{k=1}^{d} G_{k} \operatorname{diag}\left(T^{k}, 0\right)
$$

for arbitrary $G_{k} \in \mathcal{C}_{G}^{d}\left(X^{\wedge}, \boldsymbol{g}^{\bullet}\right)$ for $k=0, \ldots, d$.

Analogous notation will be used for the case of discrete asymptotics; the corresponding space of operators is then denoted by $\mathcal{C}_{G}^{d}\left(X^{\wedge}, \boldsymbol{g}^{\bullet}\right)$. We call the elements of $\mathcal{C}_{G}^{d}\left(X^{\wedge}, \boldsymbol{g}\right)\left(\mathcal{C}_{G}^{d}\left(X^{\wedge}, \boldsymbol{g}^{\bullet}\right)\right)$ Green operators on the cone $X^{\wedge}$ of type $d$, with continuous (discrete) asymptotics and weight data $g$.

This definition admits a slight generalisation to asymptotic types where the weight intervals for $Q$ and $Q^{\prime}$ are $\Xi:=(\xi, 0]$ for arbitrary $-\infty \leq \xi<0$ in place of $\Theta=(\vartheta, 0],-\infty \leq \vartheta<0$, i.e., for $P, P^{\prime}$ and $Q, Q^{\prime}$ we can take independent weight intervals $\Theta$ and $\Xi$, respectively. We then say that corresponding Green operators are associated with weight data $\boldsymbol{g}:=(\gamma, \Theta ; \delta, \Xi)$. 
THEOREM 1.3. Let $G$ be a Green operators of type $d \in \mathbb{N}$, associated with weight data $\boldsymbol{g}:=(\gamma, \Theta ; \delta, \Xi)$. Then $G$ is compact as an operator

$$
\begin{aligned}
G: \mathcal{K}^{s, \gamma}\left(X^{\wedge}\right) & \oplus \mathcal{K}^{s^{\prime}, \gamma-\frac{1}{2}}\left((\partial X)^{\wedge}, \mathbb{C}^{j_{-}}\right) \\
& \rightarrow \mathcal{K}^{s, \delta}\left(X^{\wedge}\right) \oplus \mathcal{K}^{s^{\prime}, \delta-\frac{1}{2}}\left((\partial X)^{\wedge}, \mathbb{C}^{j_{+}}\right)
\end{aligned}
$$

for every $s, s^{\prime} \in \mathbb{R}, s>-\frac{1}{2}>d-\frac{1}{2}$.

Proof. From (1.11) it follows that $G$ is a continuous operator into the space

$$
\langle r\rangle^{-N} \mathcal{K}^{N, \delta+\varepsilon}\left(X^{\wedge}\right) \oplus\langle r\rangle^{-N} \mathcal{K}^{N, \delta+\varepsilon-\frac{1}{2}}\left((\partial X)^{\wedge} \mathbb{C}^{j_{+}}\right)
$$

for every $N \in \mathbb{N}$ and $\varepsilon>0, \varepsilon$ less than the distance of $\pi_{\mathbb{C}} P$ and $\pi_{\mathbb{C}} P^{\prime}$ to the weight line $\operatorname{Re} z=\frac{n+1}{2}-\delta$. The spaces (1.14) are compactly embedded into the target spaces for every sufficiently large $N$.

REMARK 1.4. Let $G$ be a Green operator on the cone $X^{\wedge}$, associated with the weight data $(\gamma, \Theta ; \delta, \Xi)$. Then

$$
\operatorname{diag}\left(r^{\beta}, r^{\beta}\right) G \operatorname{diag}\left(r^{-\alpha}, r^{-\alpha}\right)
$$

for $\alpha, \beta \in \mathbb{R}$ is a Green operator on $X^{\wedge}$, associated with the weight data $(\gamma+$ $\alpha, \Theta ; \delta+\beta, \Xi)$.

We now fix vector boundles $E, F \in \operatorname{Vect}(X), J_{-}, J_{+} \in \operatorname{Vect}(\partial X)$, and set $E, F ; \boldsymbol{v}=\left(E, F ; J_{-}, J_{+}\right)$. There is a straightforward generalisation of Green operators on $X^{\wedge}$ of type $d$ (and discrete or continuous asymptotics) acting between the respective weighted Sobolev spaces of distributional sections in the bundles $E, F$ and $J_{-}, J_{+}$. We denote the corresponding spaces of operators by

$$
\mathcal{C}_{G}^{d}\left(X^{\wedge}, \boldsymbol{g} ; \boldsymbol{v}\right) \text { and } \mathcal{C}_{G}^{d}\left(X^{\wedge}, \boldsymbol{g}^{\bullet} ; \boldsymbol{v}\right)
$$

respectively. Instead of (1.11) we then have

$$
\begin{aligned}
G: \mathcal{K}^{s, \gamma}\left(X^{\wedge}, E\right) & \oplus \mathcal{K}^{s^{\prime}, \gamma-\frac{1}{2}}\left((\partial X)^{\wedge}, J_{-}\right) \\
& \rightarrow \mathcal{S}_{P}^{\delta}\left(X^{\wedge}, F\right) \oplus \mathcal{S}_{P^{\prime}}^{\delta-\frac{1}{2}}\left((\partial X)^{\wedge}, J_{+}\right),
\end{aligned}
$$

etc.

1.2.2. Green operators in boundary value problems. The chosen measures on $X$ and $\partial X$ allow us to identify the spaces $C^{\infty}(X \times X), C^{\infty}(X \times \partial X), C^{\infty}(\partial X \times X)$ and $C^{\infty}(\partial X \times \partial X)$ with continuous operators $C_{0}^{\infty}(X) \rightarrow C^{\infty}(X), C_{0}^{\infty}(\partial X) \rightarrow$ $C^{\infty}(X)$, etc., by $u(x) \rightarrow \int_{X} c\left(x, x^{\prime}\right) u\left(x^{\prime}\right) d x^{\prime}, v(y) \rightarrow \int_{Y} d\left(x, y^{\prime}\right) v\left(y^{\prime}\right) d y^{\prime}$ for kernels $c\left(x, x^{\prime}\right) \in C^{\infty}(X \times X), d\left(x, y^{\prime}\right) \in C^{\infty}(X \times \partial X)$, etc.

Definition 1.5. Let $\mathcal{B}^{-\infty, 0}\left(X ; j_{-}, j_{+}\right)$for $j_{-}, j_{+} \in \mathbb{N}$ denote the space of all operators

$$
\mathcal{G}: \begin{gathered}
C_{0}^{\infty}(X) \\
C_{0}^{\infty}\left(\partial X, \mathbb{C}^{j_{-}}\right)
\end{gathered} \rightarrow \quad \begin{gathered}
C^{\infty}(X) \\
C^{\infty}\left(\partial X, \mathbb{C}^{j_{+}}\right)
\end{gathered}
$$


$\mathcal{G}=\left(G_{i j}\right)_{i, j=1,2}$, where $G_{11}$ has a kernel in $C^{\infty}(X \times X)$, while $G_{12}$ is a row vector (with $j_{-}$components) of operators with kernels in $C^{\infty}(X \times \partial X), G_{21}$ is a column vector (with $j_{+}$components) of operators with kernels in $C^{\infty}(\partial X \times X)$, and $G_{22}$ is a $j_{+} \times j_{-}$matrix of operators with kernels in $C^{\infty}(\partial X \times \partial X)$. Moreover, for $d \in \mathbb{N}$, the space $\mathcal{B}^{-\infty, d}\left(X ; j_{-}, j_{+}\right)$is defined to be the set of all operators

$$
\mathcal{G}=\mathcal{G}_{0}+\sum_{i=1}^{d} \mathcal{G}_{i} \operatorname{diag}\left(T^{i}, 0\right)
$$

for arbitrary $\mathcal{G}_{i} \in \mathcal{B}^{-\infty, 0}\left(X ; j_{-}, j_{+}\right), i=0, \ldots, d$.

The elements of $\mathcal{B}^{-\infty, d}\left(X ; j_{-}, j_{+}\right)$are called smoothing Green operators of type $d$ in the algebra of boundary value problems on $X$; especially the entries of the form $G_{21}$ are also called smoothing trace operators of type $d$, and $G_{12}$ smoothing potential operators.

Note that the representation of elements in $\mathcal{B}^{-\infty, d}\left(X ; j_{-}, j_{+}\right)$of the form (1.16) is not unique. An alternative unique representation (see, for instance, [9]) gives rise to a natural semi-norm system under which $\mathcal{B}^{-\infty, d}\left(X ; j_{-}, j_{+}\right)$is a nuclear Fréchet space.

If $\boldsymbol{v}=\left(E, F ; J_{-}, J_{+}\right)$is a tuple of bundles $E, F \in \operatorname{Vect}(X), J_{ \pm} \in \operatorname{Vect}(\partial X)$, we have an easy generalisation of the space of Definition 1.5 to a space $\mathcal{B}^{-\infty, d}(X ; \boldsymbol{v})$ of operators

$$
\mathcal{G}: \underset{H^{s^{\prime}}\left(\partial X, J_{-}\right)}{H^{s}(X, E)} \rightarrow C^{\infty}\left(\partial X, J_{+}\right)
$$

which are continuous for all $s, s^{\prime} \in \mathbb{R}, s>d-\frac{1}{2}$. Also this space if Fréchet, and there is then a parameter-dependent analogue

$$
\mathcal{B}^{-\infty, d}\left(X ; \boldsymbol{v} ; \mathbb{R}^{l}\right)
$$

of such operator families $\mathcal{G}(\lambda), \lambda \in \mathbb{R}^{l}$, where $(1.17)$ is defined as $\mathcal{S}\left(\mathbb{R}^{l}, \mathcal{B}^{-\infty, d}(X ; \boldsymbol{v})\right)$. A similar notation is used for the case $l=1$ when $\mathbb{R}$ is replaced by $\operatorname{Im} z$ on any 'weight line'

$$
\Gamma_{\beta}:=\{z \in \mathbb{C}: \quad \operatorname{Re} z=\beta\} .
$$

1.2.3. Mellin operators and conormal symbols. Let $\mathbf{A} \mathbf{s}^{d, \bullet}(X)$ denote the set of all sequences

$$
R=\left\{\left(r_{j}, m_{j}, L_{j}\right)\right\}_{j \in \mathbb{Z}}
$$

for $r_{j} \in \mathbb{C}, m_{j} \in \mathbb{N}$, and finite-dimensional subspaces $L \subset \mathcal{B}^{-\infty, d}(X ; \boldsymbol{v})$ of operators of finite rank, here $\pi_{\mathbb{C}} R \cap\left\{z \in \mathbb{C}: c \leq \operatorname{Re} z \leq c^{\prime}\right\}$ is a finite set for every $c \leq c^{\prime}$, where $\pi_{\mathbb{C}} R:=\left\{r_{j}\right\}_{j \in \mathbb{Z}}$.

The space $\mathcal{M}_{R}^{-\infty, d}(X ; \boldsymbol{v})$ for $R \in \mathbf{A s}^{d, \bullet}(X)$ is defined to be the set of all elements $f(z) \in \mathcal{A}\left(\mathbb{C} \backslash \pi_{\mathbb{C}} R, \mathcal{B}^{-\infty, d}(X ; \boldsymbol{v})\right)$ that are meromorphic with poles at all $r_{j}$ of multiplicity $m_{j}+1$ and Laurent coefficients at $\left(z-r_{j}\right)^{-(k+1)}$ belonging to 
$L_{j}$ for all $0 \leq k \leq m_{j}$, where for every $\pi_{\mathbb{C}} R-$ excision function $\chi(z)$ we have $\left.\chi(z) f(z)\right|_{\Gamma_{\beta}} \in \mathcal{B}^{-\infty, d}\left(X ; \boldsymbol{v} ; \Gamma_{\beta}\right)$ for every $\beta \in \mathbb{R}$, uniformly in $c \leq \beta \leq c^{\prime}$ for every $c \leq c^{\prime}$. Note that $\mathcal{M}_{R}^{-\infty, d}(X, \boldsymbol{v})$ is a Fréchet space in a canonical way.

With every $f(z) \in \mathcal{M}_{R}^{-\infty, d}(X, \boldsymbol{v})$ we can associate an operator

$$
\mathrm{op}_{M}^{\beta}(f)=M_{\beta}^{-1} f(z) M_{\beta},
$$

where $M_{\beta}$ is the weighted Mellin transform of weight $\beta \in \mathbb{R}$, i.e., $\left(M_{\beta} u\right)(z):=$ $M\left(r^{-\beta} u\right)(z+\beta)$. We are interested in the behaviour of operators (1.19) near $r=0$. Therefore, we mainly consider them in combination with cut-off functions, i.e., look at $\omega(r) r^{j} \mathrm{op}_{M}^{\beta}(f) \tilde{\omega}(r)$ for cut-off functions $\omega, \tilde{\omega}$. To normalise weights we insert $\beta=\gamma-\frac{n}{2}$ for a weight $\gamma \in \mathbb{R}$, where $n=\operatorname{dim} X$.

We now turn to the space of Green plus smoothing Mellin operators with asymptotics on the (stretched) cone $X^{\wedge}$, first for discrete and then for continuous asymptotics.

Let us fix weight data $\boldsymbol{g}:=(\gamma, \delta, \Theta)$ for $\gamma, \delta \in \mathbb{R}$ and a weight interval $\Theta:=$ $(-(k+1), 0]$ for some $k \in \mathbb{N}$.

The space $\mathcal{C}_{M+G}^{d}\left(X^{\wedge}, \boldsymbol{g}^{\bullet} ; \boldsymbol{v}\right)$ is defined as the set of all operators of the form $M+G$, where $G$ is a Green operator of type $d$ associated with $\boldsymbol{g}$, with discrete asymptotics, cf. the preceding section, while

$$
M:=r^{\gamma-\delta} \omega(r) \sum_{j=0}^{k} r^{j} \mathrm{op}_{M}^{\gamma_{j}-\frac{n}{2}}\left(f_{j}\right) \tilde{\omega}(r)
$$

for cut-off functions $\omega, \tilde{\omega}$, and elements $f_{j} \in \mathcal{M}_{R_{j}}^{-\infty, d}(X ; \boldsymbol{v})$, where the weights $\gamma_{j} \in \mathbb{R}$, satisfy the conditions (1.26) and

$$
\pi_{\mathbb{C}} R_{j} \cap \Gamma_{\frac{n+1}{2}-\gamma_{j}}=\emptyset \text { for all } j=0, \ldots, k \text {. }
$$

Proposition 1.6. Every $A \in \mathcal{C}_{M+G}^{d}\left(X^{\wedge}, g^{\bullet} ; \boldsymbol{v}\right)$ induces continuous operators

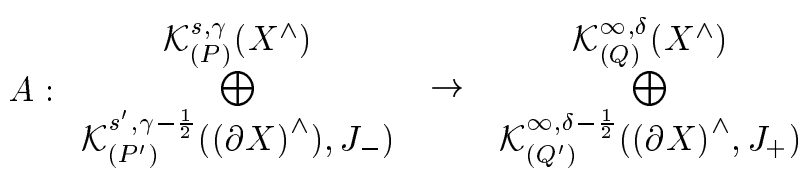

for all $s, s^{\prime} \in \mathbb{R}, s>d-\frac{1}{2}$, where subscripts ' $(P)$ ', '( $\left(P^{\prime}\right)$ ' etc. mean corresponding continuity properties for spaces without asymptotics as well as for spaces with asymptotics of type $P \in \operatorname{As}\left(X ;(\gamma, \Theta)^{\bullet}\right), P^{\prime} \in \operatorname{As}\left(\partial X ;\left(\gamma-\frac{1}{2}, \Theta\right)^{\bullet}\right)$ and resulting asymptotic types $Q \in \operatorname{As}\left(X ;(\delta, \Theta)^{\bullet}\right), Q^{\prime} \in \operatorname{As}\left(\partial X,\left(\delta-\frac{1}{2}, \Theta\right)^{\bullet}\right)$, that depend on the operator $A$ (not on $s)$.

If $A \in \mathcal{C}_{M+G}^{d}\left(X^{\wedge}, \boldsymbol{g}^{\bullet} ; \boldsymbol{v}\right)$ is written as before, we set

$$
\sigma_{M}^{\delta-\gamma-j}(A)(z):=f_{j}(z)
$$

called the conormal symbols of $A$ of order $\delta-\gamma-j, j=0, \ldots, k$. 
TheOrem 1.7. The conormal symbols of $A \in \mathcal{C}_{M+G}^{d}\left(X^{\wedge}, \boldsymbol{g}^{\bullet} ; \boldsymbol{v}\right)$ are uniquely determined by $A$. If $\tilde{A} \in \mathcal{C}_{M+G}^{d}\left(X^{\wedge}, \boldsymbol{g}^{\bullet} ; \boldsymbol{v}\right)$ is another element such that

$$
\sigma_{M}^{\delta-\gamma-j}(A)(z)=\sigma_{M}^{\delta-\gamma-j}(\tilde{A})(z)
$$

for $j=0, \ldots, k$, we have $A=\tilde{A} \bmod \mathcal{C}_{G}^{d}\left(X^{\wedge}, \boldsymbol{g}^{\bullet} ; \boldsymbol{v}\right)$.

Proof. Uniqueness of the conormal symbols in terms of the action of the operator $A$ can be proved in an analogous manner as for a manifold $X$ without boundary, cf. [6]. What remains is to show that for every fixed $j \geq 1$ and $f \in \mathcal{M}_{R}^{-\infty, d}(X ; \boldsymbol{v}), \pi_{\mathbb{C}} R \cap\left\{\Gamma_{\frac{n+1}{2}-\alpha} \cup \Gamma_{\frac{n+1}{2}-\beta}\right\}=\emptyset$ for different weights with the property $0 \leq \gamma-\alpha \leq j, 0 \leq \gamma-\beta \leq j$, we have

$$
r^{\gamma-\delta+j}\left\{\omega(r) \operatorname{op}_{M}^{\alpha-\frac{n}{2}}(f) \tilde{\omega}(r)-\sigma(r) \operatorname{op}_{M}^{\beta-\frac{n}{2}}(f) \tilde{\sigma}(r)\right\} \in \mathcal{C}_{G}^{d}\left(X^{\wedge}, \boldsymbol{g}^{\bullet} ; \boldsymbol{v}\right),
$$

where $\omega, \tilde{\omega}$ and $\sigma, \tilde{\sigma}$ are arbitrary cut-off functions. By definition the operators of type $d$ can be written as sums of operators of type zero composed with differentiations in $t$ of orders $0 \leq k \leq d$. A similar representations holds for the involved Mellin symbols. In order to get (1.23) it suffices to consider the summands separately, and to ignore the differentiations in $t$. This reduces the considerations to the case $d=0$. In this case we can proceed as in the case without boundary. First we see that a change of cut-off functions only changes our Mellin operators by Green ones, cf., similarly [9], and then it is admitted to change weights modulo Green remainders, analogously to $[\mathbf{9}]$.

Theorem 1.8. Let $A \in \mathcal{C}_{M+G}^{d}\left(X^{\wedge}, \boldsymbol{g}^{\bullet} ; \boldsymbol{v}\right)$ and $B \in \mathcal{C}_{M+G}^{e}\left(X^{\wedge}, \boldsymbol{h}^{\bullet} ; \boldsymbol{w}\right)$ for $\boldsymbol{g}:=$ $(\delta, \beta, \Theta), \boldsymbol{v}:=\left(J_{0}, J_{+}\right), \boldsymbol{h}:=(\gamma, \delta, \Theta), \boldsymbol{w}:=\left(J_{-}, J_{0}\right)$. Then $A B \in \mathcal{C}_{M+G}^{e}\left(X^{\wedge},(\boldsymbol{g} \circ\right.$ $\left.\boldsymbol{h})^{\bullet} ; \boldsymbol{v} \circ \boldsymbol{w}\right)$ for $\boldsymbol{g} \circ \boldsymbol{h}:=(\gamma, \beta, \Theta), \boldsymbol{v} \circ \boldsymbol{w}:=\left(J_{-}, J_{+}\right)$. We have for the composition the following Mellin translation formula

$$
\sigma_{M}^{\beta-\gamma-l}(A B)=\sum_{p+r=l}\left(T^{\delta-\gamma-r} \sigma_{M}^{\beta-\delta-p}(A)\right) \sigma_{M}^{\delta-\gamma-r}(A)
$$

for $l=0, \ldots, k,\left(T^{\rho} f\right)(z):=f(z+\rho)$. If $A$ or $B$ belongs to the space with subscript $G$, then the same is true of $A B$.

The calculus of operators on a manifold with edges requires an extension of Mellin symbols with discrete asymptotics to continuous asymptotics.

To every $V \in \mathcal{V}$ we want to define a space $M_{V}^{-\infty, d}(X ; \boldsymbol{v})$ of operator-valued Mellin symbols. We shall first consider the 'quasi-discrete' case, i.e., there is a sequence $\left\{K_{j}\right\}_{j \in \mathbb{Z}}$ of compact sets $K_{j} \in \mathcal{V}$ such that $\sigma_{j}:=\sup \left\{\operatorname{Re} z: z \in K_{j}\right\}$ $\left\langle\rho_{j}:=\inf \left\{\operatorname{Re} z: z \in K_{j+1}\right\}\right.$ for all $j$, and $\sigma_{j} \rightarrow-\infty$ for $j \rightarrow-\infty, \rho_{j} \rightarrow+\infty$ for $j \rightarrow+\infty$ (this refers to the case that we have an infinite sequence in both directions; we also admit $V$ to be compact or contained in a half plane $\operatorname{Re} z<\sigma$ or $\operatorname{Re} z>\rho$ for some $\sigma, \rho$; but this case is easier, so we do not discuss it separately).

As in the preceding section we consider the space $\mathcal{B}^{-\infty, d}(X ; \boldsymbol{v})$ for $\boldsymbol{v}=\left(J_{-}, J_{+}\right)$ with its Fréchet topology as well as the parameter-dependent analogue $\mathcal{B}^{-\infty, d}\left(X ; \boldsymbol{v} ; \Gamma_{\beta}\right)$, 
$\beta \in \mathbb{R}$. For every compact subset $K \subset \mathbb{C}$ we have the space $\mathcal{A}^{\prime}\left(K, \mathcal{B}^{-\infty, d}(X ; \boldsymbol{v})\right)$ of $\mathcal{B}^{-\infty, d}(X ; \boldsymbol{v})$-valued analytic functionals, carried by $K$.

Definition 1.9. The space $\mathcal{M}_{V}^{-\infty, d}(X ; \boldsymbol{v})$ for $V=\cup_{j \in \mathbb{Z}} K_{j}$ with component sets $K_{j}$ is defined to be the subspace of all $f(z) \in \mathcal{A}\left(\mathbb{C} \backslash V, \mathcal{B}^{-\infty, d}(X ; \boldsymbol{v})\right)$ such that for every $V$-excision function $\chi(z)$ we have

$$
\left.\chi(z) f(z)\right|_{\Gamma_{\beta}} \in \mathcal{B}^{-\infty, d}\left(X ; \boldsymbol{v} ; \Gamma_{\beta}\right)
$$

for every $\beta \in \mathbb{R}$, uniformly in $c \leq \beta \leq c^{\prime}$, for every $c \leq c^{\prime}$.

The definition gives rise to a canonical nuclear Fréchet topology in the space $\mathcal{M}_{V}^{-\infty, d}(X ; \boldsymbol{v})$.

Given an element $f(z) \in \mathcal{M}_{V}^{-\infty, d}(X ; \boldsymbol{v})$ we can form a sequence of analytic functionals

defined by

$$
\zeta_{j} \in \mathcal{A}^{\prime}\left(K_{j}, \mathcal{B}^{-\infty, d}(X ; \boldsymbol{v})\right), j \in \mathbb{Z},
$$

$$
\left\langle\zeta_{j}, h\right\rangle=\frac{1}{2 \pi i} \int_{C_{j}} f(z) h(z) d z, h \in \mathcal{A}(\mathbb{C}),
$$

where $C_{j}$ is a smooth counter-clockwise oriented compact curve, surrounding $K_{j}$ in a strip $\left\{z: c \leq \operatorname{Re} z \leq c^{\prime}\right\}$ that does not intersect $V \backslash K_{j}$. In this way we obtain a sequence of continuous maps

$$
\zeta_{j}: \mathcal{M}_{V}^{-\infty, d}(X ; \boldsymbol{v}) \longrightarrow \mathcal{A}^{\prime}\left(K_{j}, \mathcal{B}^{-\infty, d}(X ; \boldsymbol{v})\right), j \in \mathbb{Z} .
$$

Let now $V \in \mathcal{V}$ be an arbitrary element. Then $V$ can be written as $V=V_{1} \cup V_{2}$, where $V_{k}=\cup_{j \in \mathbb{Z}} K_{j, k}$ is quasi-discrete for $k=1,2$. We then have the Fréchet spaces $\mathcal{M}_{V_{k}}^{-\infty, d}(X ; \boldsymbol{v}), k=1,2$, and they are subspaces of $\mathcal{A}\left(\mathbb{C} \backslash V, \mathcal{B}^{-\infty, d}(X ; \boldsymbol{v})\right)$. This enables us to define

$$
\mathcal{M}_{V}^{-\infty, d}(X ; \boldsymbol{v}):=\mathcal{M}_{V_{1}}^{-\infty, d}(X ; \boldsymbol{v})+\mathcal{M}_{V_{2}}^{-\infty, d}(X ; \boldsymbol{v})
$$

as a non-direct sum of Fréchet spaces.

Proposition 1.10. The space $\mathcal{M}_{V}^{-\infty, d}(X ; \boldsymbol{v})$ for $V \in \mathcal{V}$ is independent of the specific choice of the decomposition $V=V_{1}+V_{2}$ into quasi-discrete sets.

The proof employs a (vector-valued) Cousin problem argument. Let us now fix an element $f(z) \in \mathcal{M}_{V}^{-\infty, d}(X ; \boldsymbol{v}), V \in \mathcal{V}$, and assume that $\Gamma_{\frac{n+1}{2}-\gamma_{j}} \cap V=\emptyset$ for some $\gamma_{j} \in \mathbb{R}$. Then the Mellin pseudo-differential action $\operatorname{op}_{M}^{\gamma_{j}-\frac{n^{2}}{2}}(f)$ makes sense, and similarly to (1.20) we can form operators for $j>0$ which are continuous for

$$
0 \leq \gamma-\gamma_{j} \leq j
$$

In addition the restriction to subspaces with continuous asymptotic types $P \in$ $\operatorname{As}(X,(\gamma, \Theta)), P^{\prime} \in \operatorname{As}\left(\partial X,\left(\gamma-\frac{1}{2}, \Theta\right)\right)$ induces continuous operators (1.22) for resulting asymptotic types $Q \in \operatorname{As}(X,(\gamma, \Theta)), Q^{\prime} \in \operatorname{As}\left(\partial X,\left(\gamma-\frac{1}{2}, \Theta\right)\right)$.

Let us now formulate similar structures and results for operators with continuous asymptotics. Green operators in this context have been defined in Section1.2.1. 
To define Mellin operators associated with symbols $f \in \mathcal{M}_{V}^{-\infty, d}(X ; \boldsymbol{v})$ for arbitrary $V \in \mathcal{V}$ we have to be aware that, in general, $V \cap \Gamma_{\beta}$ is not empty for arbitrary $\beta \in \mathbb{R}$. For this reason we decompose the Mellin symbol $f$ in a appropriate way, according to the following observation which is a modification of Proposition 1.10.

Remark 1.11. For every $f \in \mathcal{M}_{V}^{-\infty, d}(X ; \boldsymbol{v})$ and arbitrary reals $\beta_{1}, \beta_{2} \in \mathbb{R}$, $\beta_{1} \neq \beta_{2}$, there is a decomposition

$$
f=f_{1}+f_{2} \text { for } f_{i} \in \mathcal{M}_{V_{i}}^{-\infty, d}(X ; \boldsymbol{v}),
$$

for suitable $V_{i} \in \mathcal{V}$, such that $V_{i} \cap \Gamma_{\beta_{i}}=\emptyset, i=1,2$, where $V=V_{1}+V_{2}$

According to (1.20) and to the result of Theorem 1.7 we want to form operator $A$ with prescribed conormal symbols

$$
\sigma_{M}^{\delta-\gamma-j}(A)(z)=f_{j}(z) \in \mathcal{M}_{V_{j}}^{-\infty, d}(X ; \boldsymbol{v})
$$

for arbitrary $V_{j} \in \mathcal{V}, j=0, \ldots, k$. To obtain an analogue of the continuity (1.22) we assume

$$
V_{0} \cap \Gamma_{\frac{n+1}{2}-\gamma}=\emptyset .
$$

In general, in a composition result as in Theorem 1.8, because of the expected Mellin translation behaviour of resulting conormal symbols also in the case with continuous asymptotics, we cannot require fixed gaps in the asymptotic types $V_{j}$ for $j \geq 1$. Therefore we decompose $f_{j} \in \mathcal{M}_{V_{j}}^{-\infty, d}(X ; \boldsymbol{v})$ as

$$
f_{j}(z)=f_{1, j}(z)+f_{2, j}(z)
$$

with symbols $f_{m, j} \in \mathcal{M}_{V_{m, j}}^{-\infty, d}(X ; \boldsymbol{v})$ for $V_{m, j} \in \mathcal{V}$, such that, similarly to condition (1.21) we have

$$
V_{m, j} \cap \Gamma_{\frac{n+1}{2}-\gamma_{m, j}}=\emptyset, m=1,2,
$$

for weights $\gamma_{1, j}, \gamma_{2, j} \in \mathbb{R}$, satisfying

$$
0 \leq \gamma-\gamma_{m, j} \leq j, m=1,2 .
$$

Decompositions of the type (1.27) with these properties always exist by Remark 1.11 .

Now $\mathcal{C}_{M+G}^{d}\left(X^{\wedge}, \boldsymbol{g} ; \boldsymbol{v}\right)$ is defined as the set of all operators $A:=M_{1}+M_{2}+G$ where

$$
M_{m}:=r^{\gamma-\delta} \omega(r) \sum_{j=0}^{k} r^{j} \operatorname{op}_{M}^{\gamma_{m, j}-\frac{n}{2}}\left(f_{m, j}\right) \tilde{\omega}(r)
$$

for arbitrary $f_{m, j} \in \mathcal{M}_{V_{m, j}}^{-\infty, d}(X ; \boldsymbol{v})$ and $V_{m, j} \in \mathcal{V}, \gamma_{m, j} \in \mathbb{R}$, satisfying relations (1.28) and (1.29). The elements of $\mathcal{C}_{M+G}^{d}\left(X^{\wedge}, \boldsymbol{g} ; \boldsymbol{v}\right)$ are called (smoothing) Mellin + Green operators, of type $d$, in the cone algebra of boundary value problems on $X^{\wedge}$. Similarly as before we define the sequence of conormal symbols $\sigma_{M}^{\delta-\gamma-j}(A)(z)=$ $f_{j}(z)$, in this case with $(1.27), j=0, \ldots, k$.

Remark 1.12. For Mellin + Green operators with continuous asymptotics we have an immediate anlogue of Theorem 1.8. 


\section{Symbols with values in the cone algebra}

\subsection{Green edge symbols.}

2.1.1. Operator-valued symbols and kernel cut-off. Edge symbols with asymptotics will be introduced as operator-valued symbols that pointwise act as continuous operators between spaces of a specific structure. To start with we consider a Hilbert space $E$ that is equipped with a strongly continuous group of isomorphisms $\kappa_{\lambda}: E \rightarrow E, \lambda \in \mathbb{R}_{+}$, where $\kappa_{\lambda} \kappa_{\lambda}^{\prime}=\kappa_{\lambda \lambda^{\prime}}$ for all $\lambda, \lambda^{\prime} \in \mathbb{R}_{+}$(recall that 'strongly continuous' means that $\kappa_{\lambda} u \in C\left(\mathbb{R}_{+}, E\right)$ for every $\left.u \in E\right)$. Then $\left\{\kappa_{\lambda}\right\}_{\lambda \in \mathbb{R}_{+}}$is said to be a group action on $E$. More generally, if $E=\lim _{j \in \mathbb{N}}$ is a Fréchet space, written as a projective limit of Hilbert spaces $E_{j}$, with continuous embeddings $E_{j+1} \hookrightarrow E_{j}$ for all $j$ and a group action $\left\{\kappa_{\lambda}\right\}_{\lambda \in \mathbb{R}_{+}}$on $E_{0}$ that restricts to a group action $E_{j}$ for every $j$, we say that $\left\{\kappa_{\lambda}\right\}_{\lambda \in \mathbb{R}_{+}}$is a group action on $E$.

EXAMPLE 2.1. In our applications we have, for instance, $E=\mathcal{K}^{s, \gamma}\left(X^{\wedge}\right)$ with the group action $\kappa_{\lambda}^{(n)}: u(r, x) \rightarrow \lambda^{\frac{n+1}{2}} u(\lambda r, x)$ where $n=\operatorname{dim} X$. Similarly, for $E=\mathcal{K}^{s, \gamma}\left((\partial X)^{\wedge}\right)$ we take $\kappa_{\lambda}^{(n-1)}: v\left(r, x^{\prime}\right) \rightarrow \lambda^{\frac{n}{2}} v\left(\lambda r, x^{\prime}\right), \lambda \in \mathbb{R}_{+}$. Below we use the fact that for every $P$ there is a sequence of Hilbert spaces $E_{j}, j \in \mathbb{N}$, with continuous embeddings $E_{j+1} \hookrightarrow E_{j} \hookrightarrow \ldots E_{0}=\mathcal{K}_{P}^{0, \gamma}\left(X^{\wedge}\right)$ where $\left\{\kappa_{\lambda}\right\}_{\lambda \in \mathbb{R}_{+}}$on $E_{0}$ induces group actions on $E_{j}$ for every $j$, such that $\mathcal{S}_{P}^{\gamma}\left(X^{\wedge}\right)=\lim _{j \in \mathbb{N}} E_{j}$.

To define symbols we first consider Hilbert spaces $E$ and $\tilde{E}$ with group actions $\left\{\kappa_{\lambda}\right\}_{\lambda \in \mathbb{R}_{+}}$and $\left\{\tilde{\kappa}_{\lambda}\right\}_{\lambda \in \mathbb{R}_{+}}$, respectively. Then $S^{\mu}\left(U \times \mathbb{R}^{q} ; E, \tilde{E}\right)$ for any open set $U \subset \mathbb{R}^{p}, \mu \in \mathbb{R}$, is defined to be the subspace of all $a(y, \eta) \in C^{\infty}\left(U \times \mathbb{R}^{q}, \mathcal{L}(E, \tilde{E})\right)$ such that

$$
\left\|\tilde{\kappa}_{\langle\eta\rangle}^{-1}\left\{D_{y}^{\alpha} D_{\eta}^{\beta} a(y, \eta)\right\} \kappa_{\langle\eta\rangle}\right\|_{\mathcal{L}(E, \tilde{E})} \leq c\langle\eta\rangle^{\mu-|\beta|}
$$

for all multi-indices $\alpha \in \mathbb{N}^{p}, \alpha \in \mathbb{N}^{q}$, and all $y \in K, \eta \in \mathbb{R}^{q}$, for arbitrary $K \subset \subset U$, with constants $c=(\alpha, \beta, K)>0$. Here, as usual, $\langle\eta\rangle=\left(1+|\eta|^{2}\right)^{\frac{1}{2}}$. The best possible constants $c(\alpha, \beta, K)$ in the estimates (2.1) are a semi-norm system that turn $S^{\mu}\left(U \times \mathbb{R}^{q} ; E, \tilde{E}\right)$ to a Fréchet space. Notice that this space remains the same when we replace $\eta \rightarrow\langle y\rangle$ by any strictly positive function $\eta \rightarrow[\eta]$ in $C^{\infty}\left(\mathbb{R}^{q}\right)$ where $[\eta]=|\eta|$ for $|\eta| \geq c$ for some $c>0$.

We also have a definition of symbol spaces when $\tilde{E}$ (or both $E$ and $\tilde{E}$ ) are Fréchet spaces with group actions. In the case that $E$ is a Hilbert and $\tilde{E}=\underset{j \in \mathbb{N}}{\lim } \widetilde{E}_{j}$

a Fréchet space, we first have the symbol spaces $S^{\mu}\left(U \times \mathbb{R}^{q} ; E, \tilde{E}_{j}\right), j \in \mathbb{N}$, continuously embedded in that with respect to $\tilde{E}_{j+1}$ for all $j \geq 1$, and we then set

$$
S^{\mu}\left(U \times \mathbb{R}^{q} ; E, \tilde{E}\right)=\lim _{j \in \mathbb{N}} S^{\mu}\left(U \times \mathbb{R}^{q} ; E, \tilde{E}_{j}\right)
$$

in the Fréchet topology of the projective limit. Concerning the case that both $E$ and $\tilde{E}$ are Fréchet, see, for instance, $[\mathbf{9}]$. 
Let $S^{(\mu)}\left(U \times\left(\mathbb{R}^{q} \backslash\{0\}\right) ; E, \tilde{E}\right)$ denote the set of all $f(y, \eta) \in C^{\infty}\left(U \times\left(\mathbb{R}^{q} \backslash\right.\right.$ $\{0\}) ; \mathcal{L}(E, \tilde{E}))$ such that

$$
f(y, \lambda \eta)=\lambda^{\mu} \tilde{\kappa}_{\lambda} f(y, \eta) \kappa_{\lambda}^{-1}
$$

for all $\lambda \in \mathbb{R}_{+},(y, \eta) \in U \times\left(\mathbb{R}^{q} \backslash\{0\}\right)$.

An element $a(y, \eta) \in S^{\mu}\left(U \times \mathbb{R}^{q} ; E, \tilde{E}\right)$ is said to be classical if there are elements $a_{(\mu-j)}(y, \eta) \in S^{(\mu-j)}\left(U \times\left(\mathbb{R}^{q} \backslash\{0\}\right) ; E, \tilde{E}\right), j \in \mathbb{N}$, such that for any excision function $\chi(\eta)$ we have

$$
r_{N}(y, \eta):=a(y, \eta)-\chi(\eta) \sum_{j=0}^{N} a_{(\mu-j)}(y, \eta) \in S^{\mu-(N+1)}\left(U \times \mathbb{R}^{q} ; E, \tilde{E}\right)
$$

for all $N \in \mathbb{N}$. The homogeneous components $a_{(\mu-j)}(y, \eta)$ are uniquely determined by $a(y, \eta)$; therefore, the maps $a \rightarrow a_{(\mu-j)}$ and $a \rightarrow r_{N}$ (for a fixed choice of $\chi$ ) define operators

$$
S_{\mathrm{cl}}^{\mu}\left(U \times \mathbb{R}^{q} ; E, \tilde{E}\right) \longrightarrow S^{(\mu-j)}\left(U \times\left(\mathbb{R}^{q} \backslash\{0\}\right) ; E, \tilde{E}\right),
$$

and

$$
S_{\mathrm{cl}}^{\mu}\left(U \times \mathbb{R}^{q} ; E, \tilde{E}\right) \longrightarrow S^{\mu-(N+1)}\left(U \times \mathbb{R}^{q} ; E, \tilde{E}\right),
$$

for all $j$ and $N$. Here, subscript 'cl' indicates the space of all classical symbols. The spaces on the right of (2.3) and (2.4) are Fréchet, and we then endow $S_{\mathrm{cl}}^{\mu}(U \times$ $\left.\mathbb{R}^{q} ; E, \tilde{E}\right)$ with the Fréchet topology of the projective limit with respect to all the maps (2.3) and (2.4).

For classical symbols $a(y, \eta)$ of order $\mu$ we often set

$$
\sigma_{\Lambda}(a)(y, \eta)=a_{(\mu)}(y, \eta)
$$

which is the principal symbol of $a$ of ('twisted') homogeneity $\mu$.

Let $S_{(\mathrm{cl})}^{\mu}\left(U \times \mathbb{R}^{q} \times \mathbb{C}^{l} ; E, \tilde{E}\right)$ denote the subspace of $\mathcal{A}\left(\mathbb{C}^{l}, S_{(\mathrm{cl})}^{\mu}\left(U \times \mathbb{R}^{q} ; E, \tilde{E}\right)\right)$ such that elements $h(y, \eta, w) \in S_{(\mathrm{cl})}^{\mu}\left(U \times \mathbb{R}^{q} \times \mathbb{C}^{l} ; E, \tilde{E}\right)$ satisfy the condition $h(y, \eta, \lambda+i \tau) \in S_{(\mathrm{cl})}^{\mu}\left(U \times \mathbb{R}_{\eta, \lambda}^{q+l} ; E, \tilde{E}\right)$ for each $\tau \in \mathbb{R}^{l}$, uniformly in $\tau \in K$ for every $K \subset \subset \mathbb{R}^{l}$. The space $S_{(\mathrm{cl})}^{\mu}\left(U \times \mathbb{R}^{q} \times \mathbb{C}^{l} ; E, \tilde{E}\right)$ is Fréchet in a natural way.

Let $E$ and $\tilde{E}$ be Hilbert spaces with group action $\left\{\kappa_{\lambda}\right\}_{\lambda \in \mathbb{R}_{+}}$and $\left\{\tilde{\kappa_{\lambda}}\right\}_{\lambda \in \mathbb{R}_{+}}$, respectively, and let $g(y, \eta, \lambda) \in S_{(\mathrm{cl})}^{\mu}\left(U \times \mathbb{R}^{q+l} ; E, \tilde{E}\right)$. Set

$$
k(g)(y, \eta, \zeta):=\int e^{i \zeta \lambda} g(y, \eta, \lambda) d \lambda
$$

and

$$
H(\varphi) g(y, \eta, w):=\int e^{-i w \zeta} \varphi(\zeta) k(g)(y, \eta, \zeta) d \zeta
$$

where $\varphi(\zeta) \in C_{0}^{\infty}\left(\mathbb{R}^{l}\right), w=\lambda+i \tau \in \mathbb{C}^{l}$. For every fixed $(y, \eta) \in U \times \mathbb{R}^{q}$, we then have

$$
k(g)(y, \eta, \zeta) \in \mathcal{S}^{\prime}\left(\mathbb{R}^{l}, \mathcal{L}(E, \tilde{E})\right)=\mathcal{L}\left(\mathcal{S}\left(\mathbb{R}^{l}\right), \mathcal{L}(E, \tilde{E})\right) .
$$


It is easy to verify that

$$
\zeta^{\alpha} k(g)(y, \eta, \zeta)=k\left(\left(-D_{\lambda}^{\alpha}\right) g\right)(y, \eta, \zeta)
$$

for every multi-index $\alpha$.

Proposition 2.2. For every $\varphi \in C_{0}^{\infty}\left(\mathbb{R}^{l}\right)$ and fixed $g \in S_{(\mathrm{cl})}^{\mu}\left(U \times \mathbb{R}^{q+l} ; E, \tilde{E}\right)$ we have

$$
H(\varphi) g(y, \eta, \lambda) \in S_{(\mathrm{cl})}^{\mu}\left(U \times \mathbb{R}^{q+l} ; E, \tilde{E}\right),
$$

and the map $\varphi \rightarrow H(\varphi) g$ induces a continuous operator

$$
C_{0}^{\infty}\left(\mathbb{R}^{l}\right) \longrightarrow S_{(\mathrm{cl})}^{\mu}\left(U \times \mathbb{R}^{q+l} ; E, \tilde{E}\right) .
$$

The proof is based on generalities on the symbol spaces and is left to the reader.

Proposition 2.3. Let $\varphi \in C_{0}^{\infty}\left(\mathbb{R}^{l}\right)$ and assume $\varphi(\zeta) \equiv 1$ in a neighborhood of $\zeta=0$. Then we have $H(\varphi) g(y, \eta, w) \in S_{(\mathrm{cl})}^{\mu}\left(U \times \mathbb{R}^{q} \times \mathbb{C}^{l} ; E, \tilde{E}\right)$ and $H(\varphi) g(y, \eta, \lambda)=$ $g(y, \eta, \lambda) \bmod S^{-\infty}\left(U \times \mathbb{R}^{q+l} ; E, \tilde{E}\right)$.

Proof. We have

$$
g_{-\infty}(y, \eta, \lambda):=F_{\zeta \rightarrow \lambda}(1-\varphi(\zeta)) k(g)(y, \eta, \lambda) \in S^{-\infty}\left(U \times \mathbb{R}^{q+l} ; E, \tilde{E}\right),
$$

and

$$
g(y, \eta, \lambda)=F_{\zeta \rightarrow \lambda} k(g)(y, \eta, \lambda)=F_{\zeta \rightarrow \lambda} \varphi(\zeta) k(g)(y, \eta, \lambda)+g_{-\infty}(y, \eta, \lambda)
$$

gives us that

$$
H(\varphi) g(y, \eta, \lambda)=F_{\zeta \rightarrow \lambda} \varphi(\zeta) k(g)(y, \eta, \lambda)=g(y, \eta, \lambda)-g_{-\infty}(y, \eta, \lambda)
$$

which belongs to $S_{(\mathrm{cl})}^{\mu}\left(U \times \mathbb{R}^{q+l} ; E, \tilde{E}\right)$. This yields

$$
H(\varphi) g(y, \eta, \lambda)=g(y, \eta, \lambda) \bmod S^{-\infty}\left(U \times \mathbb{R}^{q+l} ; E, \tilde{E}\right) .
$$

To show $H(\varphi) g(y, \eta, w) \in S_{(\mathrm{cl})}^{\mu}\left(U \times \mathbb{R}^{q} \times \mathbb{C}^{l} ; E, \tilde{E}\right)$ we first observe

$$
H(\varphi) g(y, \eta, w) \in \mathcal{A}\left(\mathbb{C}_{w}^{l}, S_{(\mathrm{cl})}^{\mu}\left(U \times \mathbb{R}^{q} ; E, \tilde{E}\right)\right) .
$$

For $w=\lambda+i \tau$ we obtain

$$
\begin{aligned}
H(\varphi) g(y, \eta, w)=\int e^{-i w \zeta} \varphi(\zeta) & k(g)(y, \eta, \zeta) d \zeta \\
& =\int e^{-i \lambda \zeta} e^{\tau \zeta} \varphi(\zeta) k(g)(y, \eta, \zeta) d \zeta .
\end{aligned}
$$

Applying Proposition 2.2 to the family of functions $e^{\tau \zeta} \varphi(\zeta)$ in place of $\varphi(\zeta)$ we see that

$$
H(\varphi) g(y, \eta, \lambda+i \tau) \in S_{(\mathrm{cl})}^{\mu}\left(U \times \mathbb{R}^{q+l} ; E, \tilde{E}\right)
$$

for every fixed $\tau$ and uniformly in $\tau \in K$ for every $K \subset \subset \mathbb{R}^{l}$. 
Let $\varphi(\zeta)$ be given as in Proposition 2.3. We want to derive an asymptotic expansion of $H(\varphi) g(y, \eta, \lambda+i \tau)$ in terms of $g(y, \eta, \lambda)$ for every $\tau \in \mathbb{R}^{l}$. To this end we write the right hand side of (2.7) in the form

$$
\begin{array}{r}
H(\varphi) g(y, \eta, \lambda+i \tau)=\int e^{-i \lambda \zeta} \sum_{k=0}^{M} \frac{1}{k !}(\tau \zeta)^{k} \varphi(\zeta) k(g)(y, \eta, \zeta) d \zeta \\
+\int e^{-i \lambda \zeta} \psi_{M}(\zeta) k(g)(y, \eta, \zeta) d \zeta
\end{array}
$$

where $\psi_{M}(\zeta)=\varphi(\zeta)\left(e^{\tau \zeta}-\sum_{k=0}^{M} \frac{1}{k !}(\tau \zeta)^{k}\right) \in C_{0}^{\infty}\left(\mathbb{R}^{l}\right)$. For every $N \in \mathbb{N}$ we can choose $M$ so large that $\varphi_{M}(\zeta):=|\zeta|^{-2 N} \psi_{M}(\zeta) \in C_{0}^{\infty}\left(\mathbb{R}^{l}\right)$. The second term on the right of (2.8) takes the form

$$
\begin{aligned}
\int e^{-i \lambda \zeta} \varphi_{M}(\zeta)|\zeta|^{2 N} k(g)(y, \eta, \zeta) d \zeta \\
=\int e^{-i \lambda \zeta} \varphi_{M}(\zeta) k(g)\left(\Delta_{\lambda}^{N} g\right)(y, \eta, \zeta) d \zeta,
\end{aligned}
$$

cf. the formula (2.6). Using of $\Delta_{\lambda}^{N} g \in S_{(\mathrm{cl})}^{\mu-2 N}\left(U \times \mathbb{R}^{q+l} ; E, \tilde{E}\right)$ we obtain from Proposition 2.2 that the right hand side of $(2.9)$ belongs to $S_{(\mathrm{cl})}^{\mu-2 N}\left(U \times \mathbb{R}^{q+l} ; E, \tilde{E}\right)$.

To characterise the first term on the right of $(2.8)$ we write

$$
\frac{1}{k !}(\tau \zeta)^{k}=\alpha_{k}(\tau) \beta_{k}(\zeta)
$$

where $\alpha_{k}(\tau)$ and $\beta_{k}(\zeta)$ are corresponding homogeneous polynomials of order $k$. Then

$$
\begin{aligned}
H(\varphi) g(y, \eta, w) & =\sum_{k=0}^{M} \alpha_{k}(\tau) \int e^{-i \lambda \zeta} \varphi(\zeta) k\left(\beta_{k}\left(-D_{\lambda}\right) g\right)(y, \eta, \zeta) d \zeta \\
& =\sum_{k=0}^{\infty} \alpha_{k}(\tau) H(\varphi)\left(\beta_{k}\left(-D_{\lambda}\right) g\right)(y, \eta, \lambda)
\end{aligned}
$$

We then have $\beta_{k}\left(-D_{\lambda}\right) g(y, \eta, \lambda) \in S_{(\mathrm{cl})}^{\mu-k}\left(U \times \mathbb{R}^{q+l} ; E, \tilde{E}\right)$. By the first part of the proof this is equal to $H(\varphi)\left(\beta_{k}\left(-D_{\lambda}\right) g\right) \bmod S^{-\infty}\left(U \times \mathbb{R}^{q+l} ; E, \tilde{E}\right)$ for all $k$. This shows the following corollaries.

Corollary 2.4. We have

$$
H(\varphi) g(y, \eta, \lambda+i \tau) \sim \sum_{k=0}^{\infty} \alpha_{k}(\tau) \beta_{k}\left(-D_{\lambda}\right) g(y, \eta, \lambda)
$$

in the space $S_{(\mathrm{cl})}^{\mu}\left(U \times \mathbb{R}^{q+l} ; E, \tilde{E}\right)$ for every $\tau \in \mathbb{R}^{l}$, where the polynomials $\alpha_{k}(\tau)$ and $\beta_{k}(\tau)$ are defined by the formula (2.10). 
Corollary 2.5. If $\varphi(\zeta) \in C_{0}^{\infty}\left(\mathbb{R}^{l}\right)$ is a cut-off function, i.e., $\varphi(\zeta) \equiv 1$ in a neighbourhood of $\zeta=0$, we have

$$
\sigma_{\Lambda}^{\mu}(g)(y, \eta, \lambda)=\sigma_{\Lambda}^{\mu}(H(\varphi)(g))(y, \eta, \lambda) .
$$

Theorem 2.6. Let $h(y, \eta, w) \in S_{(\mathrm{cl})}^{\mu}\left(U \times \mathbb{R}^{q} \times \mathbb{C}^{l} ; E, \tilde{E}\right)$. Then for every $\tau_{0}, \tau_{1} \in$ $\mathbb{R}^{l}$ there are constants $c_{\alpha}\left(\tau_{0}, \tau_{1}\right), \alpha \in \mathbb{N}^{l}$, such that

$$
h\left(y, \eta, \lambda+i \tau_{1}\right) \sim \sum_{\alpha \in \mathbb{N}^{l}} c_{\alpha}\left(\tau_{0}, \tau_{1}\right) D_{\lambda}^{\alpha} h\left(y, \eta, \lambda+i \tau_{0}\right)
$$

where $c_{0}\left(\tau_{0}, \tau_{1}\right)=1$.

Proof. For every $\varphi \in C_{0}^{\infty}\left(\mathbb{R}^{l}\right)$ with $\varphi(\zeta) \equiv 1$ near $\zeta=0$ we have

$$
d(y, \eta, \lambda):=h(y, \eta, \lambda)-H(\varphi) h(y, \eta, \lambda) \in S^{-\infty}\left(U \times \mathbb{R}^{q+l} ; E, \tilde{E}\right),
$$

cf. the proof of Proposition 2.3. Applying Corollary 2.4 to $d(y, \eta, \lambda)$ we obtain $d(y, \eta, \lambda+i \tau) \in S^{-\infty}\left(U \times \mathbb{R}^{q+l} ; E, \tilde{E}\right)$ for every $\tau \in \mathbb{R}^{l}$. Again from Corollary 2.4 it follows that

$$
\begin{aligned}
h(y, \eta, \lambda+i \tau)=H(\varphi) & h(y, \eta, \lambda+i \tau)+d(y, \eta, \lambda+i \tau) \\
& \sim \sum_{k=0}^{\infty} \alpha_{k}(\tau) \beta_{k}\left(-D_{\lambda}\right) h(y, \eta, \lambda) .
\end{aligned}
$$

Applying this for $\tilde{h}(y, \eta, \lambda):=h\left(y, \eta, \lambda+i \tau_{0}\right)$ we obtain

$$
\tilde{h}\left(y, \eta, \lambda+i \tau_{1}\right) \sim \sum_{k=0}^{\infty} \alpha_{k}\left(\tau_{1}\right) \beta_{k}\left(-D_{\lambda}\right) \tilde{h}(y, \eta, \lambda)
$$

which just means our assertion.

2.1.2. Green symbols. We now turn to a specific class of operator-valued symbols, the so-called Green symbols which are in the pseudo-differential calculus on a manifold with edges responsible for a part of asymptotics

of solutions to elliptic equations. At the same time the symbols of extra trace and potential operators may formally be treated as Green symbols. For that reason we shall introduce them as $3 \times 3$ block matrices $g(y, \eta)=\left(g_{m k}(y, \eta)\right)_{m, k=1,2,3}$ continuous operators

$$
\begin{array}{ccc}
\mathcal{K}^{s, \gamma}\left(X^{\wedge}\right) & \mathcal{K}^{\infty, \delta}\left(X^{\wedge}\right) \\
\left.\left(g_{m k}(y, \eta)\right)_{m, k=1,2,3}: \mathcal{K}^{s^{\prime}, \gamma-\frac{1}{2}}\left((\partial X)^{\wedge}\right), \mathbb{C}^{j_{-}}\right) & \rightarrow & \bigoplus^{\infty, \delta-\frac{1}{2}}\left((\partial X)^{\wedge}, \mathbb{C}^{j_{+}}\right) \\
\bigoplus_{\mathbb{C}^{l_{-}}} & \bigoplus_{\mathbb{C}^{l_{+}}}
\end{array}
$$

$g(y, \eta) \in U \times \mathbb{R}^{q}$, for given weights $\gamma, \delta \in \mathbb{R}$ and dimensions $j_{ \pm}$, where $j_{+}$corresponds to the number of trace symbols and $j_{-}$to the number of potential symbols. 
Instead of the spaces $\mathcal{K}^{\infty, \delta}\left(X^{\wedge}\right)$ in (2.12) we will take subspaces with asymptotics. To this end we define

$$
\mathcal{S}_{P}^{\delta}\left(X^{\wedge}\right):=\left\{\omega u+(1-\omega) v: u \in \mathcal{K}_{P}^{\infty, \delta}\left(X^{\wedge}\right), v \in \mathcal{S}\left(\mathbb{R}, C^{\infty}(X)\right)\right\}
$$

for any asymptotic type $P \in \operatorname{As}(X, \boldsymbol{g})$ or $P \in \operatorname{As}\left(X, \boldsymbol{g}^{\bullet}\right)$, with weight data $\boldsymbol{g}=$ $(\delta, \Theta)$. A similar observation holds the spaces $\mathcal{S}_{P^{\prime}}^{\delta^{\prime}}\left((\partial X)^{\wedge}\right)$ on the boundary, $P^{\prime} \in$ $\operatorname{As}(\partial X, \boldsymbol{g})$ or $P^{\prime} \in \operatorname{As}\left(\partial X, \boldsymbol{g}^{\bullet}\right)$. We will use the fact that there is a sequence of Hilbert spaces $\left\{E_{j}\right\}_{j \in \mathbb{N}}$ with continuous embeddings $E_{j+1} \hookrightarrow E_{j} \hookrightarrow \ldots \hookrightarrow$ $E_{0}=\mathcal{K}^{0, \delta}\left(X^{\wedge}\right)$ for all $j$, such that $\left\{\kappa_{\lambda}^{(n)}\right\}_{\lambda \in \mathbb{R}_{+}}$restricts to a group action $E_{j}$ for all $j$. On direct sums of the kind $\mathcal{K}^{s, \gamma}\left(X^{\wedge}\right) \oplus \mathcal{K}^{s-\frac{1}{2}, \gamma-\frac{1}{2}}\left((\partial X)^{\wedge}\right) \oplus \mathbb{C}^{j-}$ or $\mathcal{S}_{P}^{\delta}\left(X^{\wedge}\right) \oplus \mathcal{S}_{P^{\prime}}^{\delta-\frac{1}{2}}\left((\partial X)^{\wedge}\right) \oplus \mathbb{C}^{j_{+}}$we take the group action $\operatorname{diag}\left\{\kappa_{\lambda}^{(n)}, \kappa_{\lambda}^{(n-1)}, \operatorname{id}\right\}_{\lambda \in \mathbb{R}_{+}}$ with 'id' being the identity in the respective finite-dimensional spaces.

Definition 2.7. $\mathcal{R}_{G}^{\mu, 0}\left(U \times \mathbb{R}^{q}, \boldsymbol{g} ; \boldsymbol{w}\right)$ for $\mu \in \mathbb{R}, U \subseteq \mathbb{R}^{p}$ open, and $\boldsymbol{g}=$ $(\gamma, \delta, \Theta), \boldsymbol{w}=\left(j_{-}, j_{+} ; l_{-}, l_{+}\right)$denotes the space of all $C^{\infty}$ operator families $(2.12)$ such that $g_{0}(y, \eta):=\operatorname{diag}\left(1,\langle\eta\rangle^{-\frac{1}{2}},\langle\eta\rangle^{-\frac{n+1}{2}}\right) g(y, \eta) \operatorname{diag}\left(1,\langle\eta\rangle^{\frac{1}{2}},\langle\eta\rangle^{\frac{n+1}{2}}\right)$ satisfies the following relations

$$
g_{0}(y, \eta) \in S_{\mathrm{cl}}^{\mu}\left(U \times \mathbb{R}^{q} ; E \oplus \mathbb{C}^{l_{-}}, \mathcal{S} \oplus \mathbb{C}^{l_{+}}\right),
$$

and

$$
g_{0}^{*}(y, \eta) \in S_{\mathrm{cl}}^{\mu}\left(U \times \mathbb{R}^{q} ; \tilde{E} \oplus \mathbb{C}^{l_{+}}, \tilde{S} \oplus \mathbb{C}^{l_{-}}\right),
$$

where the pointwise adjoints are taken as in Definition 1.2, and

$$
E:=\mathcal{K}^{s, \gamma}\left(X^{\wedge}\right) \oplus \mathcal{K}^{s^{\prime}, \gamma-\frac{1}{2}}\left((\partial X)^{\wedge}, \mathbb{C}^{j_{-}}\right), S:=\mathcal{S}_{P}^{\delta}\left(X^{\wedge}\right) \oplus \mathcal{S}_{P^{\prime}}^{\delta-\frac{1}{2}}\left((\partial X)^{\wedge}, \mathbb{C}^{j_{+}}\right),
$$

and

$\tilde{E}:=\mathcal{K}^{s,-\delta}\left(X^{\wedge}\right) \oplus \mathcal{K}^{s^{\prime},-\delta+\frac{1}{2}}\left((\partial X)^{\wedge}, \mathbb{C}^{j+}\right), \tilde{S}:=\mathcal{S}_{Q}^{-\gamma}\left(X^{\wedge}\right) \oplus \mathcal{S}_{Q^{\prime}}^{-\gamma+\frac{1}{2}}\left((\partial X)^{\wedge}, \mathbb{C}^{j-}\right)$,

for all $s, s^{\prime} \in \mathbb{R}, s>-\frac{1}{2}$, with certain asymptotic types $P \in \operatorname{As}(X,(\delta, \Theta)), P^{\prime} \in$ $\operatorname{As}\left(\partial X,\left(\delta-\frac{1}{2}, \Theta\right)\right), Q \in \operatorname{As}(X,(-\gamma, \Theta)), Q^{\prime} \in \operatorname{As}\left(\partial X,\left(-\gamma-\frac{1}{2}, \Theta\right)\right)$.

More generally, $\mathcal{R}_{G}^{\mu, d}\left(U \times \mathbb{R}^{q}, \boldsymbol{g} ; \boldsymbol{w}\right)$, for $d \in \mathbb{N}$ denotes the space of all operator families $g(y, \eta)=g_{0}(y, \eta)+\sum_{j=1}^{d} g_{j}(y, \eta) \operatorname{diag}\left(T^{j}, 0,0\right)$ for arbitrary $g_{j}(y, \eta) \in$ $\mathcal{R}_{G}^{\mu-j, 0}\left(U \times \mathbb{R}^{q}, \boldsymbol{g} ; \boldsymbol{w}\right), j=0, . ., d$ where $T^{j}$ is of the same meaning as in the beginning, cf. formula (1.16).

The elements of $\mathcal{R}_{G}^{\mu, d}\left(U \times \mathbb{R}^{q}, \boldsymbol{g} ; \boldsymbol{w}\right)$ are called Green symbols of type $d$, with continuous asymptotics. By replacing the asymptotic types $P, P^{\prime}, Q, Q^{\prime}$ in Definition 2.7 by corresponding discrete ones, cf. the notation in Section 1.2.2, we obtain a narrower class of Green symbols which we denote by $\mathcal{R}_{G}^{\mu, d}\left(U \times \mathbb{R}^{q}, \boldsymbol{g}^{\bullet} ; \boldsymbol{w}\right)$.

Let

$$
\mathcal{R}_{G}^{\mu, d}\left(U \times \mathbb{R}^{q}, \boldsymbol{g} ; \boldsymbol{w}\right)_{P, Q}
$$


denote the subspace of all Green symbols with given pairs of asymptotic types $P=$ $\left(P_{1}, P_{2}\right) \in \operatorname{As}(X,(\delta, \Theta)) \times \operatorname{As}\left(\partial X,\left(\delta-\frac{1}{2}, \Theta\right)\right)$ and $Q=\left(Q_{1}, Q_{2}\right) \in \operatorname{As}(X,(-\gamma, \Theta)) \times$ $\operatorname{As}\left(\partial X,\left(-\gamma-\frac{1}{2}, \Theta\right)\right)$. We then have

$$
\mathcal{R}_{G}^{\mu, d}\left(U \times \mathbb{R}^{q}, \boldsymbol{g} ; \boldsymbol{w}\right)=\bigcup_{P, Q} \mathcal{R}_{G}^{\mu, d}\left(U \times \mathbb{R}^{q}, \boldsymbol{g} ; \boldsymbol{w}\right)_{P, Q}
$$

A similar notation may be used for pairs of corresponding discrete asymptotic types. In future, for simplicity, we will formulate relations and results for continuous asymptotics. If we say nothing other, the discrete case is completely analogous and will tacitly be used if necessary.

Remark 2.8. The spaces (2.16) are Fréchet in a canonical way.

Remark 2.9. Differentiations $D_{y}^{\alpha} D_{\eta}^{\beta}$ induce continuous operators

$$
D_{y}^{\alpha} D_{\eta}^{\beta}: \mathcal{R}_{G}^{\mu, d}\left(U \times \mathbb{R}^{q} ; \boldsymbol{g} ; \boldsymbol{w}\right)_{P, Q} \rightarrow \mathcal{R}_{G}^{\mu-|\beta|, d}\left(U \times \mathbb{R}^{q} ; \boldsymbol{g} ; \boldsymbol{w}\right)_{P, Q}
$$

for all $\alpha \in \mathbb{N}^{p}, \beta \in \mathbb{N}^{q}$.

Proposition 2.10. Let $g_{j}(y, \eta) \in \mathcal{R}_{G}^{\mu-j, d}\left(U \times \mathbb{R}^{q} ; \boldsymbol{g} ; \boldsymbol{w}\right)_{P, Q}, j \in \mathbb{N}$, be an arbitrary sequence. Then there is an asymptotic sum

$$
g \sim \sum_{j=0}^{\infty} g_{j} \text { in } \mathcal{R}_{G}^{\mu, d}\left(U \times \mathbb{R}^{q} ; \boldsymbol{g} ; \boldsymbol{w}\right)_{P, Q},
$$

i.e., for every $N \in \mathbb{N}$ we have

$$
g-\sum_{j=0}^{N} g_{j} \in \mathcal{R}_{G}^{\mu-(N+1), d}\left(U \times \mathbb{R}^{q} ; \boldsymbol{g} ; \boldsymbol{w}\right)_{P, Q},
$$

and $g$ is unique $\bmod \mathcal{R}_{G}^{-\infty, d}\left(U \times \mathbb{R}^{q} ; \boldsymbol{g} ; \boldsymbol{w}\right)_{P, Q}$.

Let $\mathcal{R}_{G}^{\mu, d}\left(U \times \mathbb{R}^{q} \times \mathbb{C}^{l} ; \boldsymbol{g} ; \boldsymbol{w}\right)_{P, Q}$ denote the space of all $f(w) \in \mathcal{A}\left(\mathbb{C}^{l}, \mathcal{R}_{G}^{\mu, d}(U \times\right.$ $\left.\left.\mathbb{R}^{q} ; \boldsymbol{g} ; \boldsymbol{w}\right)_{P, Q}\right)$ such that

$$
f(\lambda+i \tau) \in \mathcal{R}_{G}^{\mu, d}\left(U \times \mathbb{R}_{\eta, \lambda}^{q+l} ; \boldsymbol{g} ; \boldsymbol{w}\right)_{P, Q}
$$

for every $\tau \in \mathbb{R}^{l}$, uniformly in $\tau \in K$ for every $K \subset \subset \mathbb{R}^{l}$.

The space $\mathcal{R}_{G}^{\mu, d}\left(U \times \mathbb{R}^{q} \times \mathbb{C}^{l} ; \boldsymbol{g} ; \boldsymbol{w}\right)_{P, Q}$ is Fréchet in a canonical way.

Next we consider symbols which holomorphically depend on complex parameters. The general notion is as follows. Let $E$ and $\tilde{E}$ be Hilbert spaces with group actions $\left\{\kappa_{\lambda}\right\}_{\lambda \in \mathbb{R}_{+}}$and $\left\{\tilde{\kappa_{\lambda}}\right\}_{\lambda \in \mathbb{R}_{+}}$, respectively; then $S_{(\mathrm{cl})}^{\mu}\left(U \times \mathbb{R}^{q} \times \mathbb{C}^{l} ; E, \tilde{E}\right)$ denotes the subspace of all $f(w) \in \mathcal{A}\left(\mathbb{C}^{l}, S_{(\mathrm{cl})}^{\mu}\left(U \times \mathbb{R}^{q} ; E, \tilde{E}\right)\right.$ such that $f(\lambda+i \tau) \in$ $S_{(\mathrm{cl})}^{\mu}\left(U \times \mathbb{R}_{\eta, \lambda}^{q+l} ; E, \tilde{E}\right)$ for every $\tau \in \mathbb{R}^{l}$, uniformly in $\tau \in K$ for every $K \subset \subset \mathbb{R}^{l}$.

In order to specify this definition to Green symbols we first observe that there is another equivalent formulation of Definition 2.7 without order reducing factors in the $\eta$-variables. In fact, those factors have the only effect that the entries $g_{i j}$ of $g$ have different orders $\mu_{i j}$, e.g., $\mu_{11}=\mu, \mu_{12}=\mu-\frac{1}{2}, \mu_{13}=\mu-\frac{n+1}{2}$, etc. The 
equivalent definition of $g$ now consists of a system of conditions for the entries separately, for instance, for $g_{12}$ that

$$
\begin{gathered}
g_{12}(y, \eta) \in S_{\mathrm{cl}}^{\mu-\frac{1}{2}}\left(U \times \mathbb{R}^{q} ; \mathcal{K}^{s, \gamma}\left(X^{\wedge}\right), \mathcal{S}_{P^{\prime}}^{\delta-\frac{1}{2}}\left(\left(\partial X^{\wedge}\right)\right),\right. \\
g_{12}^{*}(y, \eta) \in S_{\mathrm{cl}}^{\mu-\frac{1}{2}}\left(U \times \mathbb{R}^{q} ; \mathcal{K}^{s^{\prime},-\delta-\frac{1}{2}}\left((\partial X)^{\wedge}\right), \mathcal{S}_{Q}^{-\gamma}\left(\left(X^{\wedge}\right)\right),\right.
\end{gathered}
$$

for all $s, s^{\prime} \in \mathbb{R}, s>-\frac{1}{2}$. This allows us to extend the notion of Green symbols (first for type 0 and then for arbitrary type $d$ ) to the case of covariables $(\eta, \lambda) \in \mathbb{R}^{q} \times \mathbb{C}^{l}$, i.e., we have spaces of the form

$$
\mathcal{R}_{G}^{\mu, d}\left(U \times \mathbb{R}^{q} \times \mathbb{C}^{l} ; \boldsymbol{g} ; \boldsymbol{w}\right)_{P, Q} .
$$

THEOREM 2.11. For every $g(y, \eta, \lambda) \in \mathcal{R}_{G}^{\mu, d}\left(U \times \mathbb{R}_{\eta, \lambda}^{q+l} ; \boldsymbol{g} ; \boldsymbol{w}\right)_{P, Q}$ there exists an $h(y, \eta, w) \in \mathcal{R}_{G}^{\mu, d}\left(U \times \mathbb{R}_{\eta}^{q} \times \mathbb{C}_{w}^{l} ; \boldsymbol{g} ; \boldsymbol{w}\right)_{P, Q}$ such that

$$
g(y, \eta, \lambda)-h(y, \eta, \lambda) \in \mathcal{R}_{G}^{-\infty, d}\left(U \times \mathbb{R}_{\eta, \lambda}^{q+l} ; \boldsymbol{g} ; \boldsymbol{w}\right)_{P, Q},
$$

and $h(y, \eta, w)$ is unique $\bmod \mathcal{R}_{G}^{-\infty, d}\left(U \times \mathbb{R}_{\eta, \lambda}^{q+l} ; \boldsymbol{g} ; \boldsymbol{w}\right)_{P, Q}$.

Proof. Without loss of generality we assume $d=0$; otherwise we apply the arguments for the factors at $\operatorname{diag}\left(T^{j}, 0,0\right)$ separately. Let us choose an arbitrary function $\psi(\zeta) \in C_{0}^{\infty}\left(\mathbb{R}^{l}\right)$ such that $\psi(\zeta)=1$ in a neighborhood of $\zeta=0$. Then

$$
H(\psi) g(y, \eta, w):=\int e^{-i w \zeta} \psi(\zeta) k(g)(y, \eta, \zeta) d \zeta
$$

is holomorphic in the variable $w=\lambda+i \tau \in \mathbb{C}^{l}$. To analyse the nature of $H(\psi) g$ we go back to the definition. The Green symbol $g$ is a $3 \times 3$ block matrix $\left(g_{i j}\right)_{i, j=1,2,3}$ of operator-valued symbols $g_{i j}$. Let us consider, for instance, $g_{11}$; the other entries can be treated in an analogous manner. To simplify notation we write $g$ instead of $g_{11}$ which is an element of $S_{(\mathrm{cl})}^{\mu}\left(U \times \mathbb{R}_{\eta, \lambda}^{q+l} ; \mathcal{K}^{s, \gamma}\left(X^{\wedge}\right), \mathcal{S}_{P}^{\delta}\left(X^{\wedge}\right)\right)$. Setting $E^{s}:=\mathcal{K}^{s, \gamma}\left(X^{\wedge}\right)$ for fixed $s>-\frac{1}{2}$ and writing $\mathcal{S}_{P}^{\delta}\left(X^{\wedge}\right)=\lim _{j \in \mathbb{N}} \tilde{E}_{j}$ with spaces $\tilde{E}_{j}$ where $\left\{\kappa_{\lambda}\right\}_{\lambda \in \mathbb{R}_{+}}$ act as strongly continuous groups of isomorphisms for all $j$, we have to interpret $g$ as an element of $S_{(\mathrm{cl})}^{\mu}\left(U \times \mathbb{R}_{\eta, \lambda}^{q+l} ; E^{s}, \tilde{E}_{j}\right)$ for all $s>-\frac{1}{2}$ and all $j$. The formal adjoint $g^{*}$ can be treated in an analogous manner, i.e., the assertion is reduced to Proposition 2.3.

Theorem 2.12. Let $h(y, \eta, w) \in \mathcal{R}_{G}^{\mu, d}\left(U \times \mathbb{R}^{q} \times \mathbb{C}^{d} ; \boldsymbol{g} ; \boldsymbol{w}\right)_{P, Q}$. Then for every $\tau_{0}, \tau_{1} \in \mathbb{R}^{l}$ we have

$$
h\left(y, \eta, \lambda+i \tau_{0}\right)-h\left(y, \eta, \lambda+i \tau_{1}\right) \in \mathcal{R}_{G}^{\mu-1, d}\left(U \times \mathbb{R}^{q} \times \mathbb{C}^{l} ; \boldsymbol{g} ; \boldsymbol{w}\right)_{P, Q},
$$

and there are constants $c_{\alpha}\left(\tau_{0}, \tau_{1}\right), \alpha \in \mathbb{N}^{l}$, where $c_{0}\left(\tau_{0}, \tau_{1}\right)=1$ such that

$$
h\left(y, \eta, \lambda+i \tau_{1}\right) \sim \sum_{\alpha \in \mathbb{N} l} c_{\alpha}\left(\tau_{0}, \tau_{1}\right) D_{\lambda}^{\alpha} h\left(y, \eta, \lambda+i \tau_{0}\right) .
$$


Corollary 2.13. Let $h(y, \eta, w) \in \mathcal{R}_{G}^{\mu, d}\left(U \times \mathbb{R}^{q} \times \mathbb{C}^{l} ; \boldsymbol{g} ; \boldsymbol{w}\right)_{P, Q}$ and assume $h\left(y, \eta, \lambda+i \tau_{0}\right) \in \mathcal{R}_{G}^{\mu-1, d}\left(U \times \mathbb{R}^{q} \times \mathbb{C}^{l} ; \boldsymbol{g} ; \boldsymbol{w}\right)_{P, Q}$ for some $\tau_{0} \in \mathbb{R}^{l}$. Then we have $h(y, \eta, w) \in \mathcal{R}_{G}^{\mu-1, d}\left(U \times \mathbb{R}^{q} \times \mathbb{C}^{l} ; \boldsymbol{g} ; \boldsymbol{w}\right)_{P, Q}$.

THEOREM 2.14. Let $\varphi(\zeta) \in C_{0}^{\infty}\left(\mathbb{R}^{l}\right)$, and define $H(\varphi) g(y, \eta, w)$ for $g(y, \eta, w) \in$ $\mathcal{R}_{G}^{\mu, d}\left(U \times \mathbb{R}_{\eta, \lambda}^{q+l} ; \boldsymbol{g} ; \boldsymbol{w}\right)_{P, Q}$ by the expression $(2.5)$. Then $H(\varphi)$ induces a continuous operator

$$
H(\varphi): \mathcal{R}_{G}^{\mu, d}\left(U \times \mathbb{R}_{\eta, \lambda}^{q+l} ; \boldsymbol{g} ; \boldsymbol{w}\right)_{P, Q} \longrightarrow \mathcal{R}_{G}^{\mu, d}\left(U \times \mathbb{R}^{q} \times \mathbb{C}^{l} ; \boldsymbol{g} ; \boldsymbol{w}\right)_{P, Q} .
$$

For $h(y, \eta, w):=(H(\varphi) g)(y, \eta, w)$ and every $\tau_{0} \in \mathbb{R}^{l}$ we have asymptotic expansions

$$
h\left(y, \eta, \lambda+i \tau_{0}\right) \sim \sum_{\alpha \in \mathbb{N}^{l}} d_{\alpha}\left(\varphi, \tau_{0}\right) D_{\lambda}^{\alpha} h(y, \eta, \lambda)
$$

in $\mathcal{R}_{G}^{\mu, d}\left(U \times \mathbb{R}_{\eta, \lambda}^{q+l} ; \boldsymbol{g} ; \boldsymbol{w}\right)_{P, Q}$ with constants $d_{\alpha}\left(\varphi, \tau_{0}\right)$.

Proof. Analogously as in the preceding proof we may assume $d=0$. Again we consider, for instance, upper left corners. Then the assertion reduces to the case of operator-valued symbols in the abstract set-up of Section 2.1.1, and the result is consequence of Theorem 2.6

\subsection{Mellin edge symbols.}

2.2.1. Basic properties. There is another interesting class of operator-valued symbols for edge boundary value problems, namely Mellin symbols with asymptotics. Let us first consider discrete asymptotics.

In the following definition we set $\boldsymbol{g}=(\gamma, \gamma-\mu, \Theta)$ for $\Theta=(-(k+1), 0], k \in \mathbb{N}$, and $\boldsymbol{v}:=\left(J_{-}, J_{+} ; 0,0\right)$.

Definition 2.15. $R_{M+G}^{\mu, d}\left(U \times \mathbb{R}^{q}, \boldsymbol{g}^{\bullet} ; \boldsymbol{v}\right)$ for $U \subset \mathbb{R}^{q}$ open and $\mu \in \mathbb{R}, d \in \mathbb{N}$, denotes the space of all operator families $m(y, \eta)+g(y, \eta)$ for arbitrary $g(y, \eta) \in$ $R_{G}^{\mu, d}\left(U \times \mathbb{R}^{q}, \boldsymbol{g}^{\bullet} ; \boldsymbol{v}\right)$ and

$$
m(y, \eta):=\omega(r[\eta]) r^{-\mu} \sum_{j=0}^{k} r^{j} \sum_{|\alpha| \leq j} \operatorname{op}_{M}^{\gamma_{j}-\frac{n}{2}}\left(f_{j, \alpha}\right)(y) \eta^{\alpha} \tilde{\omega}(r[\eta])
$$

for arbitrary $f_{j, \alpha}(y, z) \in C^{\infty}\left(U, \mathcal{M}_{R_{j}}^{-\infty, d}(X ; \boldsymbol{v})\right)$ with certain $R_{j} \in A s^{d, \bullet}(X)$, and weights $\gamma_{j} \in \mathbb{R}$ such that $\pi_{\mathbb{C}} R_{j} \cap \Gamma_{\frac{n+1}{2}-\gamma_{j}}=\emptyset$ and $0 \leq \gamma-\gamma_{j} \leq j$ for all $j=0, \ldots, k$. The elements of $R_{M+G}^{\mu, d}\left(U \times \mathbb{R}^{q}, \boldsymbol{g}^{\bullet} ; \boldsymbol{v}\right)$ are called Mellin + Green edge symbols of order $\mu$ and type $d$, with discrete asymptotics. set

THEOREM 2.16. Let $a(y, \eta)=m(y, \eta)+g(y, \eta) \in R_{M+G}^{\mu, d}\left(U \times \mathbb{R}^{q}, \boldsymbol{g}^{\bullet} ; \boldsymbol{v}\right)$ and

$$
a_{0}(y, \eta):=\operatorname{diag}\left(1,\langle\eta\rangle^{-\frac{1}{2}}\right) a(y, \eta) \operatorname{diag}\left(1,\langle\eta\rangle^{\frac{1}{2}}\right) .
$$

Then we have

$$
a_{0}(y, \eta) \in \mathcal{S}_{\mathrm{cl}}^{\mu}\left(U \times \mathbb{R}^{q} ; E, \tilde{E}\right)
$$


for

$$
\begin{aligned}
& E:=\mathcal{K}^{s, \gamma}\left(X^{\wedge}\right) \oplus \mathcal{K}^{s^{\prime}, \gamma-\frac{1}{2}}\left((\partial X)^{\wedge}, \mathbb{C}^{j_{-}}\right), \\
& \tilde{E}:=\mathcal{K}^{\infty, \gamma-\mu}\left(X^{\wedge}\right) \oplus \mathcal{K}^{\infty, \gamma-\mu-\frac{1}{2}}\left((\partial X)^{\wedge}, \mathbb{C}^{j+}\right),
\end{aligned}
$$

$s, s^{\prime} \in \mathbb{R}, s>-\frac{1}{2}$. Moreover, if we assume the Mellin symbol $f_{j}$ to be independent of $y \in U$, we have

$$
a_{0}(y, \eta) \in \mathcal{S}_{\mathrm{cl}}^{\mu}\left(U \times \mathbb{R}^{q} ; F, \tilde{F}\right)
$$

for

$$
\begin{aligned}
& F:=\mathcal{K}_{P}^{s, \gamma}\left(X^{\wedge}\right) \oplus \mathcal{K}_{P^{\prime}}^{s^{\prime}, \gamma-\frac{1}{2}}\left((\partial X)^{\wedge}, \mathbb{C}^{j-}\right), \\
& \tilde{F}:=\mathcal{K}_{Q}^{\infty, \gamma-\mu}\left(X^{\wedge}\right) \oplus \mathcal{K}_{Q^{\prime}}^{\infty, \gamma-\mu-\frac{1}{2}}\left((\partial X)^{\wedge}, \mathbb{C}^{j+}\right),
\end{aligned}
$$

for every pair of asymptotic types

$$
P \in \operatorname{As}\left(X,(\gamma, \Theta)^{\bullet}\right), P^{\prime} \in \operatorname{As}\left(\partial X,\left(\gamma-\frac{1}{2}, \Theta\right)^{\bullet}\right)
$$

with some resulting

$$
Q \in \operatorname{As}\left(X,(\gamma-\mu, \Theta)^{\bullet}\right), Q^{\prime} \in \operatorname{As}\left(\partial X,\left(\gamma-\mu-\frac{1}{2}, \Theta\right)^{\bullet}\right)
$$

depending on $P, P^{\prime}$ and the Mellin symbols $f_{j, \alpha}$.

Definition 2.17. $\mathcal{R}_{M+G}^{\mu, d}\left(U \times \mathbb{R}^{q}, \boldsymbol{g} ; \boldsymbol{v}\right)$ for $U \subset \mathbb{R}^{q}$ open, $\mu \in \mathbb{R}, d \in \mathbb{N}$, denotes the space of all operator families $a(y, \eta):=m(y, \eta)+g(y, \eta)$ for arbitrary $g(y, \eta) \in \mathcal{R}_{G}^{\mu, d}\left(U \times \mathbb{R}^{q}, \boldsymbol{g} ; \boldsymbol{v}\right)$ and $m(y, \eta):=m_{1}(y, \eta)+m_{2}(y, \eta)$, for

$$
m_{l}:=\omega(r[\eta]) r^{-\mu} \sum_{j=0}^{k} r^{j} \sum_{|\alpha| \leq j} \operatorname{op}_{M}^{\gamma_{l, j}-\frac{n}{2}}\left(f_{l, j}\right)(y) \eta^{\alpha} \tilde{\omega}(r[\eta])
$$

for arbitrary $f_{l, j}(y, z) \in C^{\infty}\left(U, \mathcal{M}_{V_{l, j}}^{-\infty, d}(X ; \boldsymbol{v})\right), V_{l, j} \in \mathcal{V}$, and weights $\gamma_{l, j} \in \mathbb{R}$ such that $V_{l, j} \cap \Gamma_{\frac{1}{2}-\gamma_{l, j}}=\emptyset$ and $0 \leq \gamma-\gamma_{l, j} \leq j$ for all $j=0, \ldots, k$ and $l=1,2$. The elements of $\mathcal{R}_{M+G}^{\mu, d}\left(U \times \mathbb{R}^{q}, \boldsymbol{g} ; \boldsymbol{v}\right)$ are called Mellin + Green symbols of order $\mu$ and type $d$, with continuous asymptotics.

Let us set

$$
\sigma_{M}^{\mu-j}(a)(y, z, \eta):=\sum_{|\alpha| \leq j}\left\{f_{1, j \alpha}(y, z)+f_{2, j \alpha}(y, z)\right\} \eta^{\alpha},
$$

called the conormal symbol of $a(y, \eta)$ of (conormal) order $\mu-j, j=0, \ldots, k$. Note that $\sigma_{M}^{\mu}(a)$, the principal conormal symbol, is independent of the covariable $\eta$. Moreover, define the homogeneous principal edge symbol of order $\mu$ by

$$
\sigma_{\wedge}^{\mu}(a)(y, \eta)=\sigma_{\wedge}^{\mu}(m)(y, \eta)+\sigma_{\wedge}^{\mu}(g)(y, \eta)
$$

as the principal part of $a$ as a classical operator-valued symbol. For the Green summand $g$, this was introduced before, while for $m=m_{1}+m_{2}$ we have 


$$
\sigma_{\wedge}^{\mu}\left(m_{l}\right)(y, \eta)=\omega(r[\eta]) r^{-\mu} \sum_{j=0}^{k} r^{j} \sum_{|\alpha|=j} \operatorname{op}_{M}^{\gamma_{l, j}-\frac{n}{2}}\left(f_{l, j}\right)(y) \eta^{\alpha} \tilde{\omega}(r[\eta]) .
$$

Notice that an analogous definition makes sense when $\eta \in \mathbb{R}^{q}$ is replaced by $(\eta, \lambda) \in \mathbb{R}^{q+l}$, then the corresponding $\sigma_{\wedge}^{\mu}(a)(y, \eta, \lambda)$ has the meaning of parameterdependent homogeneous principal edge symbol.

Proposition 2.18. Let $a(y, \eta), \tilde{a}(y, \eta) \in \mathcal{R}_{M+G}^{\mu, d}\left(U \times \mathbb{R}^{q}, \boldsymbol{g} ; \boldsymbol{v}\right)$ and assume that $\sigma_{M}^{\mu-j}(a)=\sigma_{M}^{\mu-j}(\tilde{a})$, for $j=0, \ldots, k$. Then we have $a-\tilde{a} \in \mathcal{R}_{G}^{\mu, d}\left(U \times \mathbb{R}^{q}, \boldsymbol{g} ; \boldsymbol{v}\right)$. In other words, the specific choice of cut-off functions, the weights $\gamma_{l, j}$, or of the function $\eta \rightarrow[\eta]$ does not affect $a(y, \eta)$, up to Green operators. A similar statement holds for the case with discrete asymptotics.

REMARK 2.19. Note in particular, that when we replace $\omega$ or $\tilde{\omega}$ in the expression $(2.17)$ by a function in $C_{0}^{\infty}\left(\mathbb{R}_{+}\right)$then the corresponding operator function is a Green symbol.

Proposition 2.20. $\mathcal{R}_{M+G}^{\mu, d}\left(U \times \mathbb{R}^{q}, \boldsymbol{g} ; \boldsymbol{v}\right)$ is a subspace of

$$
S_{\mathrm{cl}}^{\mu}\left(U \times \mathbb{R}^{q} ; H ; \tilde{H}\right)
$$

both for

$$
\begin{gathered}
H=\mathcal{K}^{s, \gamma}\left(X^{\wedge}, E\right) \oplus \mathcal{K}^{s^{\prime}, \gamma-\frac{1}{2}}\left((\partial X)^{\wedge}, J_{-}\right), \\
\tilde{H}=\mathcal{K}^{\infty, \gamma-\mu}\left(X^{\wedge}, F\right) \oplus \mathcal{K}^{\infty, \gamma-\mu-\frac{1}{2}}\left((\partial X)^{\wedge}, J_{+}\right),
\end{gathered}
$$

and

$$
\begin{gathered}
H=\mathcal{K}_{P}^{s, \gamma}\left(X^{\wedge}, E\right) \oplus \mathcal{K}_{P^{\prime}}^{s^{\prime}, \gamma-\frac{1}{2}}\left((\partial X)^{\wedge}, J_{-}\right), \\
\tilde{H}=\mathcal{K}_{Q}^{\infty, \gamma-\mu}\left(X^{\wedge}, F\right) \oplus \mathcal{K}_{Q^{\prime}}^{\infty, \gamma-\mu-\frac{1}{2}}\left((\partial X)^{\wedge}, J_{+}\right),
\end{gathered}
$$

for every pair $\left(P, P^{\prime}\right)$ of asymptotic types, with some resulting $\left(Q, Q^{\prime}\right)$; here the order $\mu$ in (2.19) refers to the group actions $\operatorname{diag}\left(\kappa_{\lambda}^{(n)}, \lambda^{\frac{1}{2}}\right)_{\lambda \in \mathbb{R}_{+}}$.

Proposition 2.21. We have

$$
D_{y}^{\alpha} D_{\eta}^{\beta} \mathcal{R}_{M+G}^{\mu}\left(U \times \mathbb{R}^{q}, \boldsymbol{g} ; \boldsymbol{v}\right) \subseteq \mathcal{R}_{M+G}^{\mu-|\beta|}\left(U \times \mathbb{R}^{q}, \boldsymbol{g} ; \boldsymbol{v}\right)
$$

for every $\alpha, \beta \in \mathbb{N}^{q}$. Moreover, $a(y, \eta) \in \mathcal{R}_{M+G}^{\mu}\left(U \times \mathbb{R}^{q}, \boldsymbol{g} ; \boldsymbol{v}\right)$ entails

$$
D_{y}^{\alpha} D_{\eta}^{\beta} a(y, \eta) \in \mathcal{R}_{G}^{\mu-|\beta|}\left(U \times \mathbb{R}^{q}, \boldsymbol{g} ; \boldsymbol{v}\right)
$$

for every $\alpha, \beta \in \mathbb{N}^{q}$ with $|\beta|>k$ (where $k$ is linked to the weight interval $\Theta=$ $(-(k+1), 0]$, involved in $\boldsymbol{g})$. 
2.2.2. Holomorphic families of Mellin plus Green symbols. We now single out suitable Fréchet subspaces in

$$
\mathcal{R}_{M+G}^{\mu, d}\left(U \times \mathbb{R}^{q}, \boldsymbol{g} ; \boldsymbol{v}\right)
$$

cf. Definition 2.17. We consider the case of continuous asymptotics; the discrete case is simpler and easily follows from the general construction. It will be convenient to normalise the choice of the weights $\gamma_{m, j}$ in the relation $(2.17)$. For $j=0$ we have necessarily $\gamma_{m, 0}=\gamma$ for $m=1,2$. Otherwise we set

$$
\gamma_{m, j}:=\gamma-\frac{m}{3} \text { for } m=1,2 \text { and } j \geq 1
$$

We employ the fact that for every $V \in \mathcal{V}$ there are elements $V_{1}, V_{2} \in \mathcal{V}$ such that

$$
V=V_{1}+V_{2} \text { and } V_{m} \cap \Gamma_{\frac{n+1}{2}-\left(\gamma-\frac{m}{3}\right)}=\emptyset \text { for } m=1,2 .
$$

Analogously as the relation (1.25) we have

$$
C^{\infty}\left(U, \mathcal{M}_{V}^{-\infty, d}(X ; \boldsymbol{v})\right)=\sum_{m=1}^{2} C^{\infty}\left(U, \mathcal{M}_{V_{m}}^{-\infty, d}(X ; v)\right)
$$

as a non-direct sum of Fréchet spaces. This implies the following result:

Proposition 2.22. Let us fix elements $V_{0}, V \in \mathcal{V}$ and such that $V_{0} \cap \Gamma_{\frac{n+1}{2}-\gamma}=$ $\emptyset$. Then $\mathcal{R}_{M+G}^{\mu, d}\left(U \times \mathbb{R}^{q}, \boldsymbol{g} ; \boldsymbol{v}\right)_{m}$ for $m=1,2$ denote the subspace of all elements $a$ in (2.20) such that

(i) $\sigma_{M}^{\mu}(a) \in C^{\infty}\left(U, \mathcal{M}_{V}^{-\infty, d}(X ; \boldsymbol{v})\right)$,

(ii) the coefficients of the polynomial $(2.18)$ belongs to $C^{\infty}\left(U, \mathcal{M}_{V_{m}}^{-\infty, d}(X ; \boldsymbol{v})\right)$ for $j \geq 1$.

By definition we then have

$$
\mathcal{R}_{M+G}^{\mu, d}\left(U \times \mathbb{R}^{q}, \boldsymbol{g} ; \boldsymbol{v}\right)=\sum_{m=1}^{2} \mathcal{R}_{M+G}^{\mu, d}\left(U \times \mathbb{R}^{q}, \boldsymbol{g} ; \boldsymbol{v}\right)_{m},
$$

and every $a$ in (2.20) belong to (2.23) for a suitable choice of the sets $V_{0}$ and $V$. We shall Fréchet topologise the summands on the right of (2.23) separately and then endow (2.20) with the topology of non-direct sum.

Let $\mathcal{P}_{m, j}(\eta)$ denote the space of all polynomials in $\eta$ of degree $j$ such that the coefficients belong to $\mathcal{M}_{V_{m}}^{-\infty, d}(X ; \boldsymbol{v})$. Proposition 2.18 then gives us linear operators

$$
\sigma_{M}^{\mu}: \mathcal{R}_{M+G}^{\mu, d}\left(U \times \mathbb{R}^{q}, \boldsymbol{g} ; \boldsymbol{v}\right)_{m} \rightarrow C^{\infty}\left(U, \mathcal{M}_{V_{0}}^{-\infty, d}(X ; \boldsymbol{v})\right),
$$

for $j=1, \ldots, k$.

$$
\sigma_{M}^{\mu-j}: \mathcal{R}_{M+G}^{\mu, d}\left(U \times \mathbb{R}^{q}, \boldsymbol{g} ; \boldsymbol{v}\right)_{m} \rightarrow \mathcal{P}_{m, j}(\eta)
$$

Let, for a moment, $F_{m}$ denote the Cartesian product of all spaces that occur on the right of (2.24); this is a Fréchet space in canonical way. Set $\sigma_{M}:=$ $\left(\sigma_{M}^{\mu-j}\right)_{j=0, \ldots, k}$. Then $(2.24)$ represents a surjective map 


$$
\sigma_{M}: R_{m} \rightarrow F_{m}
$$

for $R_{m}:=\mathcal{R}_{M+G}^{\mu, d}\left(U \times \mathbb{R}^{q}, \boldsymbol{g} ; \boldsymbol{v}\right)_{m}$. The kernel of $(2.25)$ is the space of the Green edge symbols $\mathcal{R}_{G}^{\mu, d}\left(U \times \mathbb{R}^{q}, \boldsymbol{g} ; \boldsymbol{v}\right)$ since $(2.25)$ has a right inverse, given by expression (2.17) for a fixed choice of $\omega, \tilde{\omega},[\eta]$ and $\gamma_{m, 0}=\gamma, \gamma_{m, j}=\gamma-\frac{m}{3}$ for $j \geq 1$. It follows that

Write for a moment

$$
\mathcal{R}_{M+G}^{\mu, d}\left(U \times \mathbb{R}^{q}, \boldsymbol{g} ; \boldsymbol{v}\right)_{m} \cong F_{m} \oplus \mathcal{R}_{G}^{\mu, d}\left(U \times \mathbb{R}^{q}, \boldsymbol{g} ; \boldsymbol{v}\right) .
$$

$$
\mathcal{R}_{M+G}^{\mu, d}\left(U \times \mathbb{R}^{q}, \boldsymbol{g} ; \boldsymbol{v}\right)_{P, Q ; m}:=F_{m} \oplus \mathcal{R}_{G}^{\mu, d}\left(U \times \mathbb{R}^{q}, \boldsymbol{g} ; \boldsymbol{v}\right)_{P, Q} .
$$

We then endow the space on the left with the Fréchet structure of the direct sum. Finally, we have

$$
\mathcal{R}_{M+G}^{\mu, d}\left(U \times \mathbb{R}^{q}, \boldsymbol{g} ; \boldsymbol{v}\right)_{m}=\cup_{P, Q} \mathcal{R}_{M+G}^{\mu, d}\left(U \times \mathbb{R}^{q}, \boldsymbol{g} ; \boldsymbol{v}\right)_{P, Q ; m} .
$$

Our next objective is to apply the kernel cut-off procedure to symbols with parameter $\lambda \in \mathbb{R}^{l}$ and obtain symbols depending on the complex parameter $w \in \mathbb{C}^{l}$. Let $\mathcal{R}_{M+G}^{\mu, d}\left(U \times \mathbb{R}^{q} \times \mathbb{C}^{l}, \boldsymbol{g} ; \boldsymbol{v}\right)_{P, Q ; m}$ denote the subspaces of all $f(w) \in$ $\mathcal{A}\left(\mathbb{C}^{l}, \mathcal{R}_{M+G}^{\mu, d}\left(U \times \mathbb{R}^{q}, \boldsymbol{g} ; \boldsymbol{v}\right)_{P, Q ; m}\right)$ such that $f(\lambda+i \tau) \in \mathcal{R}_{M+G}^{\mu, d}\left(U \times \mathbb{R}_{\eta, \lambda}^{q+l}, \boldsymbol{g} ; \boldsymbol{v}\right)_{P, Q ; m}$ for every $\tau \in \mathbb{R}^{l}$, uniformly in $\tau \in K$ for every $K \subset \subset \mathbb{R}^{l}$.

The space $\mathcal{R}_{M+G}^{\mu, d}\left(U \times \mathbb{R}^{q} \times \mathbb{C}^{l}, \boldsymbol{g} ; \boldsymbol{v}\right)_{P, Q ; m}$ is Fréchet in a canonical way.

Theorem 2.23. For every $a(y, \eta, \lambda) \in \mathcal{R}_{M+G}^{\mu, d}\left(U \times \mathbb{R}_{\eta, \lambda}^{q+l}, \boldsymbol{g} ; \boldsymbol{w}\right)_{P, Q ; m}$ there exists a $b(y, \eta, w) \in \mathcal{R}_{M+G}^{\mu, d}\left(U \times \mathbb{R}_{\eta}^{q} \times \mathbb{C}_{w}^{l}, \boldsymbol{g} ; \boldsymbol{w}\right)_{P, Q ; m}$ such that

$$
a(y, \eta, \lambda)-b(y, \eta, \lambda) \in \mathcal{R}_{G}^{-\infty, d}\left(U \times \mathbb{R}_{\eta, \lambda}^{q+l}, \boldsymbol{g} ; \boldsymbol{w}\right)_{P, Q}
$$

and $b(y, \eta, w)$ is unique $\bmod \mathcal{R}_{G}^{-\infty, d}\left(U \times \mathbb{R}^{q+l}, \boldsymbol{g} ; \boldsymbol{w}\right)_{P, Q}$, where

$$
\sigma_{\wedge}^{\mu}(a)(y, \eta, \lambda)=\sigma_{\wedge}^{\mu}(b)(y, \eta, \lambda) .
$$

Proof. The first steps of the proof are the same as in the proof of Theorem 2.11. We may restrict the consideration to the case $d=0$ and formally replace $g$ by our $a$. When we form $H(\psi) a(y, \eta, w)$ we obtain a holomorphic operator-valued function by the general cut-off technique of Section 2.1.1. It remains to verify that

$$
H(\psi) a(y, \eta, w) \in \mathcal{R}_{M+G}^{\mu, d}\left(U \times \mathbb{R}^{q} \times \mathbb{C}^{l}, \boldsymbol{g} ; \boldsymbol{w}\right)_{P, Q ; m}
$$

as well as the relation (2.27). From the formula (2.8) with $\psi$ and $a$ instand of $\varphi$ and $g$, we see that

$$
(H(\psi) a)(y, \eta, \lambda+i \tau)=a(y, \eta, \lambda)+b_{M}(y, \eta, \tau)+r_{M}(y, \eta, \tau)
$$

for

$$
\begin{gathered}
b_{M}(y, \eta, \tau):=\sum_{n=1}^{M} \int e^{-i \lambda \zeta} \frac{1}{n !}(\tau \zeta)^{n} \psi(\zeta) k(a)(y, \eta, \zeta) d \zeta \\
r_{M}(y, \eta, \tau):=\int e^{-i \lambda \zeta} \psi_{M}(\zeta) k(a)(y, \eta, \zeta) d \zeta
\end{gathered}
$$


As in the proof of Proposition 2.3 we can apply integrations by part in the integral of $b_{M}$ which gives us

$$
b_{M}(y, \eta, \tau)=\sum_{n=1}^{M} \alpha_{n}(\tau) \beta_{n}\left(-D_{\lambda}\right) a(y, \eta, \lambda)
$$

in the notations of Corollary 2.4, modulo an error which is of order $-\infty$ in the sense of operator-valued symbols. To see that the asymptotic sum in the sense of Corollary 2.4 is valid in our narrower class of smoothing Mellin+Green symbols of prescribed asymptotic types, it suffices to observe that for sufficiently large $M$ the difference $b_{M+1}-b_{M}$ is of Green type; i.e. for sufficiently large $M$ the remainder $r_{M}$ only contributes Green symbols, and the differentiations in $\lambda$ as they are contained in formula (2.11) to not change $P$ and $Q$ in the expression, which allows us to apply Proposition 2.10.

TheOREM 2.24. Let $a(y, \eta, \lambda) \in \mathcal{R}_{M+G}^{\mu, d}\left(U \times \mathbb{R}^{q+1} ; \boldsymbol{g}, \boldsymbol{v}\right)$ and $b(y, \eta, \lambda) \in \mathcal{R}_{M+G}^{\nu, e}(U \times$ $\left.\mathbb{R}^{q+1} ; \boldsymbol{h}, \boldsymbol{w}\right)$, for $\boldsymbol{h}:=(\gamma, \gamma-\mu, \Theta), \boldsymbol{w}:=\left(J_{-}, J_{0}\right), \boldsymbol{g}:=(\gamma-\mu, \gamma-\mu-\nu, \Theta)$, $\boldsymbol{v}:=\left(J_{0}, J_{+}\right)$. Then we have ab $\in \mathcal{R}_{M+G}^{\mu+\nu, e}\left(U \times \mathbb{R}^{q+1} ; \boldsymbol{g} \circ \boldsymbol{h}, \boldsymbol{w} \circ \boldsymbol{v}\right)$ for $\boldsymbol{g} \circ \boldsymbol{h}:=$ $(\gamma, \gamma-\mu-\nu, \Theta), \boldsymbol{w} \circ \boldsymbol{v}=\left(J_{-}, J_{+}\right)$, and $\sigma_{\wedge}^{\mu+\nu}(a b)=\sigma_{\wedge}^{\mu}(a) \sigma_{\wedge}^{\nu}(b)$.

\section{Kernel cut-off for cone operators where the base is a manifold with edge}

\subsection{Operators for corner singularities.}

3.1.1. Manifolds with edges. In this section we introduce a class of manifolds with edges and boundary. Because we mainly consider analytic aspects from operator algebras and in order to limit the burden of formalities, we content ourselves with a sufficiently simple case. In that sense a manifold $W$ with edge $Y \subset W$ and boundary is a topological space such that $W \backslash Y$ is a $C^{\infty}$ manifold with boundary, $Y$ is a compact closed $C^{\infty}$ manifold, and $Y$ has a neighbourhood $U$ in $W$ that is homeomorphic to a wedge $X^{\triangle} \times Y$, where

$$
X^{\triangle}:=\left(\overline{\mathbb{R}}_{+} \times X\right) /(\{0\} \times X)
$$

is a cone with base $X$ that is a compact $C^{\infty}$ manifold with boundary $\partial X$. In addition we assume that the homemorphism $h: V \rightarrow X^{\triangle} \times Y$ restricts to diffeomorphisms

$$
U \backslash Y \cong X^{\wedge} \times Y, U \cap Y \cong Y
$$

for $X^{\wedge}:=\mathbb{R}_{+} \times X$. In other words, in $V \backslash Y$ we fixed a global splitting of variables into $(r, x, y) \in \mathbb{R}_{+} \times X \times Y$. Other admissible splittings $(\tilde{r}, x, \tilde{y})$ are required to be related to $(r, x, y)$ by a diffeomorphism $\mathbb{R}_{+} \times X \times Y \rightarrow \mathbb{R}_{+} \times X \times Y$ (in the sense of $C^{\infty}$ manifolds with boundary) that extends to a diffeomorphism

$$
\mathbb{R} \times X \times Y \rightarrow \mathbb{R} \times X \times Y .
$$

In particular, we obtain homeomorphisms $\overline{\mathbb{R}}_{+} \times X \times Y \rightarrow \overline{\mathbb{R}}_{+} \times X \times Y$ with smoothness up to $r=0$ in the sense of (3.1), and there is then a so-called stretched manifold $\mathbb{W}$ associated with $W$ that has $\overline{\mathbb{R}}_{+} \times X \times Y$ as local model near $r=0$ 
(rather than $X^{\triangle} \times Y$ for $\mathrm{W}$ ). The space $\mathbb{W}$ is a manifold with corners of a specific kind caused by $\partial X$ (the case $\partial X=\emptyset$ is also admitted; $\mathbb{W}$ is a $C^{\infty}$ manifold with boundary $\partial \mathbb{W} \cong \partial X \times Y)$.

The space $\partial(W \backslash Y) \cup Y=: V$ is then a manifold with edge without boundary as in the definition before with $\partial X=\emptyset$.

Another exmple of such a 'closed' manifold with edge $Y$ is the double $2 W$ of $W$ which we obtain by gluing together two copies of $W \backslash Y$ along $\partial(W \backslash Y)$ which gives us $2(W \backslash Y)$ and then set $2 W=\{2(W \backslash Y)\} \cup Y$. The associated stretched manifold $2 \mathbb{W}$ is a smooth manifold with boundary $\partial(2 \mathbb{W})$, and this boundary is a $2 X$ bundle over $Y$.

Let us set

$$
\mathbb{W}_{\text {sing }}:=\partial(2 \mathbb{W}) \cap \mathbb{W}
$$

where $\mathbb{W}$ is identified with one of the copies $\mathbb{W}_{ \pm}$of $\mathbb{W}$ in $2 \mathbb{W}$, and

$$
\mathbb{W}_{\text {reg }}:=\mathbb{W} \backslash \mathbb{W}_{\text {sing }} \text {. }
$$

In a similar manner we set for the stretched manifold $\mathbb{V}$ associated with $V$

$$
\mathbb{V}_{\text {sing }}:=\partial \mathbb{V}, \quad \mathbb{V}_{\text {reg }}:=\mathbb{V} \backslash \mathbb{V}_{\text {sing }} .
$$

3.1.2. Edge spaces with asymptotics. Let us now introduce some classes of weighted spaces and subspaces with (continuous) asymptotics on a (stretched) manifold $\mathbb{W}$ with edges and boundary. It will be easier to start from the case when the base $X$ of the model cone has an empty boundary; then in order to reach the case with boundary, we first consider the double of the base which is then closed and compact and finally restrict our distributions to the given configuration (the plus-side of the double). The structure of weighted Sobolev spaces near the edge is formally linked to the constructions of Section 2.1.1 on Hilbert spaces or Fréchet spaces $E$ with group action $\left\{\kappa_{\lambda}\right\}_{\lambda \in \mathbb{R}_{+}}$.

If $E$ is first a Hilbert space, $\mathcal{W}^{s}\left(\mathbb{R}^{q}, E\right)$ for $s \in \mathbb{R}$ is defined to be the completion of $\mathcal{S}\left(\mathbb{R}^{q}, E\right)$ (the Schwartz space of $E$-valued functions) with respect to the norm

$$
\left\{\int\langle\eta\rangle^{2 s}\left\|\kappa_{\langle\eta\rangle}^{-1} \hat{u}(\eta)\right\|_{E}^{2} d \eta\right\}^{\frac{1}{2}},
$$

with $\hat{u}(\eta)$ being the Fourier transform in $\mathbb{R}^{q}$.

For the case of a Fréchet space $E$ with group action, written as a projective limit $E=\lim _{k \in \mathbb{N}} E^{k}$ of Hilbert spaces, we have by the above construction the spaces $\mathcal{W}^{s}\left(\mathbb{R}^{q}, E^{k}\right)$ with continuous embeddings

$$
\mathcal{W}^{s}\left(\mathbb{R}^{q}, E^{k+1}\right) \hookrightarrow \mathcal{W}^{s}\left(\mathbb{R}^{q}, E^{k}\right) \text { for all } k,
$$

and we then set

$$
\mathcal{W}^{s}\left(\mathbb{R}^{q}, E\right):=\lim _{k \in \mathbb{N}} \mathcal{W}^{s}\left(\mathbb{R}^{q}, E^{k}\right)
$$

in the Fréchet topology of the projective limit.

For the case of spaces on a (stretched) wedge $\overline{\mathbb{R}}_{+} \times X \times \mathbb{R}^{q}$ with edge $\mathbb{R}^{q}$ we take the spaces $E:=\mathcal{K}^{s, \gamma}\left(X^{\wedge}\right)$ or Fréchet subspaces $E:=\mathcal{K}_{P}^{s, \gamma}\left(X^{\wedge}\right)$ with 
(discrete or continuous) asymptotics of any fixed type $P$. The group action is $\left(\kappa_{\lambda} u\right)(r, x):=\lambda^{\frac{n+1}{2}} u(\lambda r, x), \lambda \in \mathbb{R}_{+}$where $n=\operatorname{dim} X$. We then have the spaces

$$
\mathcal{W}^{s}\left(\mathbb{R}^{q}, \mathcal{K}^{s, \gamma}\left(X^{\wedge}\right)\right) \text { and } \mathcal{W}^{s}\left(\mathbb{R}^{q}, \mathcal{K}_{P}^{s, \gamma}\left(X^{\wedge}\right)\right)
$$

for every $s, \gamma \in \mathbb{R}$. It can easily be verified that

$$
\mathcal{H}_{\text {comp }}^{s}\left(X^{\triangle} \times \mathbb{R}^{q}\right) \subset \mathcal{W}^{s}\left(\mathbb{R}^{q}, \mathcal{K}^{s, \gamma}\left(X^{\wedge}\right)\right) \subset \mathcal{H}_{\text {loc }}^{s}\left(X^{\triangle} \times \mathbb{R}^{q}\right)
$$

and the same for spaces with subscript $P$ in the middle. This allows us to define global spaces on a (for simplicity compact) stretched manifold $\mathbb{W}$ with edge

$$
\mathcal{W}^{s, \gamma}(\mathbb{W}) \text { and } \mathcal{W}_{P}^{s, \gamma}(\mathbb{W})
$$

respectively, (first for the case of closed cone base $\mathrm{X}$ ). The space $\mathcal{W}_{P}^{s, \gamma}(\mathbb{W})$ is define as the set of all $u \in \mathcal{H}_{\text {loc }}^{s}(\mathrm{int} \mathbb{W})$ such that $\varphi u \in \mathcal{W}^{s}\left(\mathbb{R}^{q}, \mathcal{K}_{(P)}^{s, \gamma}\left(X^{\wedge}\right)\right)$ for every $\varphi \in C^{\infty}(\mathbb{W})$ supported in a coordinate neighbourhood intersecting $\partial \mathbb{W}$ (subscript ' $(P)^{\prime}$ ' means that we are talking on spaces without or with asymptotics; definitions are valid in both cases). On the space $\mathcal{W}^{0,0}(\mathbb{W})$ we fix a scalar product $(\cdot, \cdot)$; then $(\cdot, \cdot): C_{0}^{\infty}(\mathrm{int} \mathbb{W}) \times C_{0}^{\infty}(\mathrm{int} \mathbb{W}) \longrightarrow \mathbb{C}$ extends to a non-degenerate sesquilinear pairing

for all $s, \gamma \in \mathbb{R}$

$$
(\cdot, \cdot): \mathcal{W}^{s, \gamma}(\operatorname{int} \mathbb{W}) \times \mathcal{W}^{-s,-\gamma}(\operatorname{int} \mathbb{W}) \longrightarrow \mathbb{C}
$$

Now, if $X$ is a compact manifold with boundary, we can form the double $2 X$, and the pass to the double $2 \mathbb{W}$ of our given (stretched) manifold $\mathbb{W}$ with edge and boundary; let $\mathbb{W}$ be identified with $\mathbb{W}_{+}$, cf. Section 3.1.1.

Let $\mathcal{W}_{(P)}^{s, \gamma}(2 \mathbb{W})_{0}$ denote the subspace of all $\mathcal{W}_{(P)}^{s, \gamma}(2 \mathbb{W})$ that vanish on $\mathbb{W}_{-}$. We then have isomorphisms

$$
\mathcal{W}_{(P)}^{s, \gamma}\left(\mathbb{W}_{+}\right):=\left.\mathcal{W}_{(P)}^{s, \gamma}(2 \mathbb{W})\right|_{\text {int } \mathbb{W}} \cong \mathcal{W}_{(P)}^{s, \gamma}(2 \mathbb{W}) / \mathcal{W}_{(P)}^{s, \gamma}(2 \mathbb{W})_{0}
$$

(for simplicity, asymptotic types on $2 \mathbb{W}$ and $\mathbb{W}$ are denoted by the same letter; clearly the coefficient spaces refer to $2 X$ and $X$, respectively, where the ones on $2 X$ restrict to $X$ in a natural way). This gives us the corresponding quotient topologies in the spaces $\mathcal{W}_{(P)}^{s, \gamma}\left(\mathbb{W}_{+}\right)$that are Hilbert (Fréchet) for the case without (with) asymptotics.

3.1.3. Parameter-dependent edge Green operators. Let $\mathbb{W}$ be a compact stretched manifold with edge and boundary $\mathbb{V}$, cf. the notation in Section 3.1.1, and set $\boldsymbol{v}:=\left(J_{-}, J_{+} ; L_{-}, L_{+}\right)$for vector bundles $J_{-}, J_{+} \in \operatorname{Vect}(\mathbb{V}), L_{-}, L_{+} \in \operatorname{Vect}(Y)$. Then $\mathcal{Y}_{G}^{-\infty, 0}(\mathbb{W} ; \boldsymbol{g} ; \boldsymbol{v})$ for $\boldsymbol{g}:=(\gamma, \delta, \Theta)$, for weight $\gamma, \delta \in \mathbb{R}$ and a weight interval $\Theta=(\theta, 0]$ is defined to be the space of all operators

$$
\begin{aligned}
& \mathcal{W}^{s, \gamma}(\mathbb{W}) \quad \mathcal{W}_{P}^{\infty, \delta}(\mathbb{W}) \\
& \mathcal{G}: \mathcal{W}^{s^{\prime}-\frac{1}{2}, \gamma-\frac{1}{2}}\left(\mathbb{V}, J_{-}\right) \rightarrow \mathcal{W}_{P^{\prime}}^{\infty, \delta-\frac{1}{2}}\left(\mathbb{V}, J_{+}\right), \\
& \bigoplus^{s^{\prime \prime}-\frac{n+1}{2}}\left(Y, L_{-}\right) \quad H^{\infty}\left(Y, L_{+}\right)
\end{aligned}
$$


that are continuous for all $s, s^{\prime}, s^{\prime \prime} \in \mathbb{R}, s>-\frac{1}{2}$, and some asymptotic types $P \in \operatorname{As}(X,(\delta, \Theta)), P^{\prime} \in \operatorname{As}\left(\partial X,\left(\delta-\frac{1}{2}, \Theta\right)\right)$ such that the formal adjoint defines continuous operators

$$
\begin{aligned}
& \mathcal{W}^{s,-\delta}(\mathbb{W}) \quad \mathcal{W}_{Q}^{\infty,-\gamma}(\mathbb{W}) \\
& \bigoplus \\
& \mathcal{G}^{*}: \mathcal{W}^{s^{\prime},-\delta-\frac{1}{2}}\left(\mathbb{V}, J_{+}\right) \rightarrow \mathcal{W}_{Q^{\prime}}^{\infty,-\gamma-\frac{1}{2}}\left(\mathbb{V}, J_{-}\right) \text {, } \\
& \bigoplus^{s^{\prime \prime}-\frac{n+1}{2}}\left(Y, L_{+}\right) \quad H^{\infty}\left(Y, L_{-}\right)
\end{aligned}
$$

for all $s, s^{\prime}, s^{\prime \prime} \in \mathbb{R}, s>-\frac{1}{2}$, and some asymptotic types $Q \in \operatorname{As}(X,(-\gamma, \Theta))$, $Q^{\prime} \in \operatorname{As}\left(\partial X,\left(-\gamma-\frac{1}{2}, \Theta\right)\right)$. Moreover, define $\mathcal{Y}_{G}^{-\infty, d}(\mathbb{W} ; \boldsymbol{g} ; \boldsymbol{v})$ for $d \in \mathbb{N}$ to be the space of all operators of the form

$$
\mathcal{G}=\mathcal{G}_{0}+\sum_{j=1}^{d} \mathcal{G}_{j} \operatorname{diag}\left(T^{j}, 0,0\right),
$$

for arbitrary $\mathcal{G}_{j} \in \mathcal{Y}_{G}^{-\infty, d}(\mathbb{W} ; \boldsymbol{g} ; \boldsymbol{v})$ and $T$ any differential operator of first order on $\mathbb{W}$ that is represented by a vector field transversal to the boundary $\mathbb{V}$.

Finally, let $\mathcal{Y}_{G}^{-\infty, d}\left(\mathbb{W} ; \boldsymbol{g} ; \boldsymbol{v} ; \mathbb{R}^{l}\right)$ denote the space of all Schwartz functions on $\mathbb{R}^{l}$ with values in $\mathcal{Y}_{G}^{-\infty, d}(\mathbb{W} ; \boldsymbol{g} ; \boldsymbol{v})$ which refers to natural Fréchet topologies in the subspaces with fixed $P, P^{\prime}$ and $Q, Q^{\prime}$.

We now pass to Green operators on $\mathbb{W}$ with a local symbolic structure as in Definition 2.7. We define $\mathcal{Y}_{G}^{\mu, 0}\left(\mathbb{W} ; \boldsymbol{g} ; \boldsymbol{v} ; \mathbb{R}^{l}\right)$ as operator families

$$
\mathcal{G}(\lambda)=\mathcal{G}_{0}(\lambda)+\mathcal{C}(\lambda)
$$

for arbitrary $\mathcal{C}(\lambda) \in \mathcal{Y}_{G}^{-\infty, 0}\left(\mathbb{W} ; \boldsymbol{g} ; \boldsymbol{v} ; \mathbb{R}^{l}\right)$ and operators $\mathcal{G}_{0}(\lambda)$ that are locally near $\mathbb{W}_{\text {sing }}$ given as $\mathrm{Op}_{y}(g)(\lambda)$ for symbols

$$
g(y, \eta, \lambda) \in \mathcal{R}^{\mu, 0}\left(\Omega \times \mathbb{R}_{\eta, \lambda}^{q+l} ; \boldsymbol{g} ; \boldsymbol{w}\right)
$$

(with $j_{ \pm}$and $l_{ \pm}$being the fibre dimensions of $J_{ \pm}$and $L_{ \pm}$, respectively, and $\Omega \subseteq \mathbb{R}^{q}$ an open set, corresponding to local coordinates on Y). Finally, the space $\mathcal{Y}_{G}^{\mu, d}\left(\mathbb{W} ; \boldsymbol{g} ; \boldsymbol{v} ; \mathbb{R}^{l}\right)$ of parameter-dependent Green operators of type $d$ is defined as the set of all operator families of the form (3.5) for arbitrary $\mathcal{G}_{j}(\lambda) \in$ $\mathcal{Y}_{G}^{\mu-j, 0}\left(\mathbb{W} ; \boldsymbol{g} ; \boldsymbol{v} ; \mathbb{R}^{l}\right)$. There is also a natural notion of holomorphic dependence of operator families $\mathcal{G}(w)=\mathcal{G}_{0}(w)+\mathcal{C}(w)$, on $w \in \mathbb{C}^{l}$, by requiring the local amplitude functions of $\mathcal{G}(w)$ to belong to $\mathcal{R}_{G}^{\mu, d}\left(\Omega \times \mathbb{R}_{\eta}^{q} \times \mathbb{C}^{l} ; \boldsymbol{g} ; \boldsymbol{w}\right)$ for fixed asymptotic types $P, P^{\prime}$ and $Q, Q^{\prime}$ and the smoothing family $\mathcal{C}(w)$ to be holomorphic as a function with values in operators of the form (3.3) such that the pointwise adjoints take values in operator of the form (3.4) and is such a way that $\mathcal{C}(\lambda+i \tau)$ for every $\tau \in \mathbb{R}^{l}$ is a Schwartz function in $\lambda \in \mathbb{R}^{l}$ with values in such operators uniformly in compact subsets with respect to $\tau \in \mathbb{R}^{l}$. Let $\mathcal{Y}_{G}^{\mu, d}\left(\mathbb{W} ; \boldsymbol{g} ; \boldsymbol{v} ; \mathbb{C}^{l}\right)$ denote the corresponding spaces of operator functions $\mathcal{G}(\Omega)$.

\subsection{Meromorphic corner symbols.}


3.2.1. Mellin plus Green operators on a manifold with edges. Let $\mathbb{W}$ and $\mathbb{V}$ be as in Section 3.1.3. We then define the space

$$
\mathcal{Y}_{M+G}^{\mu, d}\left(\mathbb{W} ; \boldsymbol{g} ; \boldsymbol{v} ; \mathbb{R}^{l}\right)
$$

for $\boldsymbol{g}=(\gamma, \gamma-\mu, \Theta), \Theta:=(-(k+1), 0]$, as the set of all operator families

$$
\mathcal{A}(\lambda):=\mathcal{M}(\lambda)+\mathcal{G}(\lambda)
$$

with arbitrary $\mathcal{G}(\lambda) \in \mathcal{Y}_{G}^{\mu, d}\left(\mathbb{W} ; \boldsymbol{g} ; \boldsymbol{v} ; \mathbb{R}^{l}\right)$ and $\mathcal{M}(\lambda)$ being a $2 \times 2$ block matrix family

$$
\mathcal{M}(\lambda): \quad \begin{aligned}
& \mathcal{W}^{s, \gamma}(\mathbb{W}) \\
& \mathcal{W}^{s-\frac{1}{2}, \gamma-\frac{1}{2}}\left(\mathbb{V}, J_{-}\right)
\end{aligned} \rightarrow \mathcal{W}^{\infty, \gamma-\mu-\frac{1}{2}\left(\mathbb{V}, J_{+}\right)}
$$

that is locally near $\mathbb{W}_{\text {sing }}$ given as $\mathrm{Op}_{y}(m)(\lambda)$ for $m(y, \eta, \lambda) \in \mathcal{R}_{M+G}^{\mu, d}\left(\Omega \times \mathbb{R}_{\eta, \lambda}^{q+l} ; \boldsymbol{g} ; \boldsymbol{w}\right)$ for $w=\left(J_{-}, J_{+}\right), \Omega \subseteq \mathbb{R}^{q}$ open. Every $\mathcal{A}(\lambda) \in \mathcal{Y}_{M+G}^{\mu, d}\left(\mathbb{W} ; \boldsymbol{g} ; \boldsymbol{v} ; \mathbb{R}^{l}\right)$ has an invariantly defined parameter-dependent homogeneous edge symbol $\sigma_{\wedge}^{\mu}(\mathcal{A})(y, \eta, \lambda)$ that is which locally on $\Omega \times\left(\mathbb{R}^{q+l} \backslash\{0\}\right)$ which an operator family

$$
\begin{array}{ccc}
\mathcal{K}^{s, \gamma}\left(X^{\wedge}\right) & \mathcal{K}^{\infty, \gamma-\mu}\left(X^{\wedge}\right) \\
\left.\sigma_{\wedge}^{\mu}(\mathcal{A})(y, \eta, \lambda): \mathcal{K}^{s-\frac{1}{2}, \gamma-\frac{1}{2}}\left((\partial X)^{\wedge}\right), J_{-}\right) & \rightarrow & \bigoplus^{\infty, \gamma-\frac{1}{2}-\mu}\left((\partial X)^{\wedge}, J_{+}\right) \\
\bigoplus_{\mathbb{C}^{l_{-}}} & \bigoplus_{\mathbb{C}^{l_{+}}},
\end{array}
$$

given by corresponding edge symbols of amplitude functions $m(y, \eta, \lambda) \in \mathcal{R}_{M+G}^{\mu, d}(\Omega \times$ $\left.\mathbb{R}_{\eta, \lambda}^{q+l} ; \boldsymbol{g} ; \boldsymbol{w}\right)$. There is then a family of subordinate conormal symbols

$$
\sigma_{M}^{\mu}(\mathcal{A})(y, \eta, \lambda): \underset{\mathcal{K}^{s-\frac{1}{2}}\left(\partial X, J_{-}\right)}{\mathcal{H}^{s}(X)} \rightarrow \bigoplus_{\mathcal{K}^{\infty}\left(\partial X, J_{+}\right)}^{\bigoplus^{\infty}(X)}
$$

$y \in Y, z \in \Gamma_{\frac{n+1}{2}-\gamma}$. The conormal symbols are uniquely determined by $\sigma_{\wedge}^{\mu}(\mathcal{A})$.

Theorem 3.1. Let $\mathcal{A} \in \mathcal{Y}_{M+G}^{\mu, d}\left(\mathbb{W} ; \boldsymbol{g} ; \boldsymbol{v} ; \mathbb{R}^{l}\right)$ and $\mathcal{B} \in \mathcal{Y}_{M+G}^{\nu, e}\left(\mathbb{W} ; \boldsymbol{h} ; \boldsymbol{w} ; \mathbb{R}^{l}\right)$ for $\boldsymbol{g}:=(\gamma-\nu, \gamma-\nu-\mu, \Theta), \boldsymbol{v}:=\left(J_{0}, J_{+} ; L_{0}, L_{+}\right), \boldsymbol{h}:=(\gamma, \gamma-\nu, \Theta), \boldsymbol{w}:=$ $\left(J_{-}, J_{0} ; L_{-}, L_{0}\right)$. Then we have $\mathcal{A B} \in \mathcal{Y}_{M+G}^{\mu+\nu, e}(\mathbb{W},(\boldsymbol{g} \circ \boldsymbol{h}) ; \boldsymbol{v} \circ \boldsymbol{w})$, and $\sigma_{\wedge}^{\mu+\nu}(\mathcal{A B})=$ $\sigma_{\wedge}^{\mu}(\mathcal{A}) \sigma_{\wedge}^{\nu}(\mathcal{B})$

3.2.2. Kernel cut-off and holomorphic representatives. The definition of $\mathcal{Y}_{M+G}^{\mu, d}\left(\mathbb{W} ; \boldsymbol{g} ; \boldsymbol{v} ; \mathbb{R}^{l}\right)$ can be modified to a space

$$
\mathcal{Y}_{M+G}^{\mu, d}\left(\mathbb{W} ; \boldsymbol{g} ; \boldsymbol{v} ; \mathbb{C}^{l}\right)
$$

of operator functions $\mathcal{A}(w)=\mathcal{M}(w)+\mathcal{G}(w)+\mathcal{C}(w)$ where the local amplitude functions $a(y, \eta, w)$ belong to $\mathcal{R}_{M+G}^{\mu, d}\left(\Omega \times \mathbb{R}_{\eta}^{q} \times \mathbb{C}_{w}^{l} ; \boldsymbol{g} ; \boldsymbol{w}\right)$ and the smoothing family $\mathcal{C}(w)$ to $\mathcal{Y}_{G}^{-\infty, d}\left(\mathbb{W} ; \boldsymbol{g} ; \boldsymbol{v} ; \mathbb{C}^{l}\right)$. 
ThEOREM 3.2. For every $\mathcal{A}(\lambda) \in \mathcal{Y}_{M+G}^{\mu, d}\left(\mathbb{W} ; \boldsymbol{g} ; \boldsymbol{v} ; \mathbb{R}^{l}\right)$ there exists a $\mathcal{B}(w) \in$ $\mathcal{Y}_{M+G}^{\mu, d}\left(\mathbb{W} ; \boldsymbol{g} ; \boldsymbol{v} ; \mathbb{C}^{l}\right)$ such that

$$
\mathcal{A}(\lambda)-\mathcal{B}(\lambda) \in \mathcal{Y}_{G}^{-\infty, d}\left(\mathbb{W} ; \boldsymbol{g} ; \boldsymbol{v} ; \mathbb{R}^{l}\right),
$$

and $\mathcal{B}(w)$ is unique $\bmod \mathcal{Y}_{G}^{-\infty, d}\left(\mathbb{W} ; \boldsymbol{g} ; \boldsymbol{v} ; \mathbb{C}^{l}\right)$. Moreover, we have

$$
\sigma_{\wedge}^{\mu}(\mathcal{B})(y, z)=\sigma_{\wedge}^{\mu}(\mathcal{A})(y, z)
$$

Proof. We employ the fact that our operator families $\mathcal{A}(\lambda)$ (modulo families of order $-\infty)$, are locally given in the form $\mathrm{Op}_{y}(a)(\lambda)$ for an amplitude function $a(y, \eta, \lambda) \in \mathcal{R}_{M+G}^{\mu, d}\left(\Omega \times \mathbb{R}_{\eta, \lambda}^{q+l} ; \boldsymbol{g} ; \boldsymbol{w}\right), \Omega \in \mathbb{R}^{q}$ open, corresponding to a chart on the edge $Y$. Then it suffices to apply Theorem 2.23 and to replace $a(y, \eta, \lambda)$ by $b(y, \eta, w):=H(\psi)(y, \eta, w)$ for any cut-off function $\psi(\zeta)$. To show (3.6) we may apply Theorem 2.23 which gives us

$$
a(y, \eta, \lambda)=b(y, \eta, \lambda) \bmod \mathcal{R}_{M+G}^{\mu-1, d}\left(\Omega \times \mathbb{R}_{\eta, \lambda}^{q+l} ; \boldsymbol{g} ; \boldsymbol{w}\right) .
$$

This implies equality of the principal edge symbol of order $\mu$.

3.2.3. Meromorphic functions with iterated asymptotics. Let us apply the construction of the preceding section to the case $l=1$ and $\boldsymbol{g}=(\gamma, \gamma, \Theta), \boldsymbol{v}=$ $(J, J ; L, L)$ for some $J \in \operatorname{Vect}(\mathbb{V})$. Consider an element, $h(w) \in \mathcal{Y}_{M+G}^{\mu, d}(\mathbb{W} ; \boldsymbol{g} ; \boldsymbol{v} ; \mathbb{C})$ and look at the family of operators

$$
\begin{array}{ccc}
\mathcal{W}^{s, \gamma}(\mathbb{W}) & \mathcal{W}^{s, \gamma}(\mathbb{W}) \\
\bigoplus^{s-\frac{1}{2}, \gamma-\frac{1}{2}}(\mathbb{V}, J) & \rightarrow & \bigoplus^{s-\frac{1}{2}, \gamma-\frac{1}{2}}(\mathbb{V}, J) \\
\bigoplus^{s-\frac{n+1}{2}}(Y, L) & \bigoplus^{s-\frac{n+1}{2}}(Y, L)
\end{array}
$$

where 1 is the identity operator in the respective spaces, $s>d-\frac{1}{2}$.

THEOREM 3.3. Assume that the operator function (3.7) is elliptic in the sense that

$$
\begin{array}{ccc}
\mathcal{K}^{s, \gamma}\left(X^{\wedge}\right) & \mathcal{K}^{s, \gamma}\left(X^{\wedge}\right) \\
\left.\sigma_{\wedge}^{\mu}(1+h)(y, \eta, \lambda): \mathcal{K}^{s-\frac{1}{2}, \gamma-\frac{1}{2}}\left((\partial X)^{\wedge}\right), J\right) & \rightarrow \mathcal{K}^{s-\frac{1}{2}, \gamma-\frac{1}{2}}\left((\partial X)^{\wedge}, J\right), \\
\bigoplus_{\mathbb{C}^{l}}^{\bigoplus} & \bigoplus_{\mathbb{C}^{l}}
\end{array}
$$

is an isomorphism for all $(y, \eta, \lambda) \in\left(T^{*} Y \times \mathbb{R}^{l}\right) \backslash 0$. Then there exists a countable discrete set $D \subset \mathbb{C}$ with finite intersection $D \cap\left\{c \leq \operatorname{Im} w \leq c^{\prime}\right\}$ for every $c \leq c^{\prime}$, such that (3.7) is invertible for every $w \in \mathbb{C} \backslash D$ and all $s>d-\frac{1}{2}$.

PRoOF. The operators (3.7) form a holomorphic family of continuous operators between the respective Hilbert spaces. In addition $\sigma_{\wedge}^{\mu}(1+h)(y, \eta, \lambda)$ can be inverted in the sense that there are locally near $Y$ elements $p(y, \eta, \lambda) \in \mathcal{R}_{M+G}^{-\mu}(\Omega \times$ $\left.\mathbb{R}^{q} ; \boldsymbol{g} ; \boldsymbol{v}\right)\left(\Omega \subseteq \mathbb{R}^{q}\right.$ open, corresponding to charts on $\left.Y\right)$ such that $\sigma_{\wedge}^{-\mu}(1+p)(y, \eta, \lambda)$ 
are inverses of $\sigma_{\wedge}^{\mu}(1+h)(y, \eta, \lambda)$. The amplitude functions associated with $p$ give rise to a global operator function $1+P_{0}(\lambda)$ such that $\left(1+P_{0}(\lambda)\right)(1+h(\lambda))=1-R(\lambda)$, where $R(\lambda) \in \mathcal{Y}_{M+G}^{-1}(\mathbb{W} ; \boldsymbol{g} ; \boldsymbol{v} ; \mathbb{R})$. Then a formal Neumann series argument yields a $P_{1}(\lambda) \in \mathcal{Y}_{M+G}^{-\mu}(\mathbb{W} ; \boldsymbol{g} ; \boldsymbol{v} ; \mathbb{R})$ defined by

$$
1+P_{1}(\lambda)=\left\{\sum_{j=0}^{\infty} R_{j}(\lambda)\right\}\left(1+P_{0}(\lambda)\right)
$$

with $\{\ldots\}$ being a corresponding asymptotic sum, such that $\left(1+P_{1}(\lambda)\right)(1+h(\lambda))=$ $1 \bmod \mathcal{Y}^{-\infty}(\mathbb{W} ; \boldsymbol{g} ; \boldsymbol{v} ; \mathbb{R})$. Note that the asymptotic sum is admissible in the sense of Proposition 2.10 concerning the involved asymptotic types. Then, since the remainder in $\mathcal{Y}^{-\infty}(\mathbb{W} ; \boldsymbol{g} ; \boldsymbol{v} ; \mathbb{R})$ behaves like a Schwartz function in $\lambda$, the operator family $1+h(\lambda)$ becomes invertible for large $|\lambda|$. For any fixed $\lambda$ the operators are Fredholm between the respective Hilbert spaces, because the remainders which are produced pointwise are Green operators and as such compact operators. The same is true for the unique extensions to $w \in \mathbb{C}$. Now we can apply a well known theorem on the behaviour of the inverse of a holomorphic Fredholm function which gives us a meromorphic function with poles at most in a set $D$ as claimed.

Operator functions in $\mathcal{Y}_{M+G}^{\mu, d}(\mathbb{W} ; \boldsymbol{g} ; \boldsymbol{v} ; \mathbb{C})$ are part of the corner conormal symbolic structure for boundary value problems in domains or on manifolds with corner singularities, where the base is a manifold $W$ with boundary $V$ and edge $Y$ (recall that $\mathbb{W}$ and $\mathbb{V}$ in our notation are the corresponding stretched manifolds). Corresponding differential operators in a stretched corner $\mathbb{R}_{+} \times \mathbb{W}$ (with $t \in \mathbb{R}_{+}$ being the corner axis) have the form

$$
A=t^{-\mu} \sum_{j=0}^{\mu} a_{j}(t)\left(-t \frac{\partial}{\partial t}\right)^{j},
$$

with coefficients $a_{j}(t)$ that are assumed to be smooth in $t$ (up to $t=0$ ) and take values in edge-degenerate differential operators on $\mathbb{W}$ of order $\mu-j$ that are locally near $\mathbb{W}_{\text {sing }}$ in the splitting of variables into $(r, x, y) \in \mathbb{R}_{+} \times X \times \Omega, \Omega \subseteq \mathbb{R}^{q}$ open, of the form

$$
r^{(-\mu+j)} \sum_{k+|\beta| \leq \mu-j} b_{j ; k \beta}(r, y)\left(-r \frac{\partial}{\partial r}\right)^{k}\left(r D_{y}\right)^{\beta}
$$

with coefficients $b_{j ; k \beta}(r, y)$, smooth in $r, y$ (up to $r=0$ ) and with values in $\operatorname{Diff}^{\mu-j-(k-|\beta|)(X)}$, where $X$, the base of the model cone for $W$ is a compact $C^{\infty}$ manifold with boundary. Consider, for simplicity, the case that the coefficients $a_{j}$ in (3.8) are independent of $t$. Then

$$
h(w):=\sum_{j=0}^{\mu} a_{j} w_{j}
$$


is a (non-smooting) holomorphic operator-valued Mellin symbol, and we can pass to weighted Mellin operators

$$
\operatorname{op}_{M}^{\delta-\frac{1+n+q}{2}}(h): \mathcal{H}^{s, \gamma ; \delta}\left(\mathbb{W}^{\wedge}\right) \longrightarrow \mathcal{H}^{s-\mu, \gamma-\mu ; \delta}\left(\mathbb{W}^{\wedge}\right)
$$

$\mathbb{W}^{\wedge}:=\mathbb{R}_{+} \times \mathbb{W}$ operating between corner spaces in a similar sense as the ones in the beginning in Section 1.1 with $X$ in place of $W$. In the present case we have two weights, namely $\gamma, \delta \in \mathbb{R}$, where $\gamma$ belongs to the inner cone axis variable $r \in \mathbb{R}_{+}$ and $\delta$ to the corner axis variable $t \in \mathbb{R}_{+}$. The spaces $\mathcal{H}^{s, \gamma ; \delta}\left(\mathbb{W}^{\wedge}\right)$ are defined in a similar manner as the ones in [7] for the analogous case of $\mathbb{B}$ instead of $\mathbb{W}$, where $\mathbb{B}$ is a (stretched) manifold with conical singularities (formally, it corresponds to the case of a space $\mathbb{W}$ with $q=\operatorname{dim} Y=0)$. Comparing (3.9) and (3.8) we see that

$$
A=t^{-\mu} \operatorname{op}_{M}^{\delta-\frac{1+n+q}{2}}(h) \text {. }
$$

Under ellipticity assumptions on $A$ (cf. [7] and [10] for the case without boundary) and adding boundary operators of analogous corner-degenerate form shape as (3.8), we can construct parametrices of the corresponding elliptic boundary value problem on $\mathbb{W}^{\wedge}$ near $t=0$. A typical part of the parametrix consists of operators of the form

$$
t^{\mu} \operatorname{op}_{M}^{\delta-\frac{1+n+q}{2}}(f)
$$

for a suitable element $f(w):=\tilde{f}(i w)$,

$$
\tilde{f}(w) \in \mathcal{Y}_{M+G}^{\mu, d}(\mathbb{W} ; \boldsymbol{g} ; \boldsymbol{v} ; \mathbb{C}) .
$$

Operators of the form (3.10) are the contribution to Green's function of the corresponding elliptic boundary value problem that generates (iterated) corner asymptotics of solutions. (Because of this structure we might better speak of Green's plus Mellin's function). As a result of the parametrix construction one concludes iterated corner asymptotics of solutions $u(t)$ (taking values in spaces with edge asymptotics on $\mathbb{W}$ ), that are reflected by meromorphy of the Mellin transform of $u(t)$ in the covariable $w \in \mathbb{C}$. The nature of such corner asymptotics for the case without boundary was analysed in [10]. The present results, in particular, the analysis of meromorphic corner conormal symbols, play a similar role in boundary value problems on the ones in the boundaryless case. The full description of the parametrix constructions needs a certain conterpart of our paper that just deels with non-smoothing Mellin operators with holomorphic symbols, cf. Oliaro and Schulze [4]. The algebra generated by the present calculus together with that of [4] will be investigated elswhere. It will contain the parametrices of elliptic boundary value problems on manifolds with corners where the corner bases are manifolds with edges.

\section{References}

[1] L. Boutet de Monvel, Boundary problems for pseudo-differential operators, Acta Math. 126 (1971), 11-51. 
[2] D. Kapanadze and B.-W. Schulze, Crack theory and edge singularities, Kluwer Academic Publ., Dordrecht, 2003, (to appear).

[3] V.A. Kondratyev, Boundary value problems for elliptic equations in domains with conical points, Trudy Mosk. Mat. Obshch. 16 (1967), 209-292.

[4] A. Oliaro and B.-W. Schulze, Parameter-dependent boundary value problems on manifolds with edges, Preprint 2002/25, Institut für Mathematik, Potsdam, 2002.

[5] B.-W. Schulze, Regularity with continuous and branching asymptotics for elliptic operators on manifolds with edges, Integral Equations Operator Theory 11 (1988), 557-602.

[6] B.-W. Schulze, Pseudo-differential operators on manifolds with singularities, NorthHolland, Amsterdam, 1991.

[7] B.-W. Schulze, The Mellin pseudo-differential calculus on manifolds with corners, Symposium: "Analysis in Domains and on Manifolds with Singularities", Breitenbrunn 1990, Teubner-Texte zur Mathematik, vol. 131, Teubner, Leipzig, 1992, pp. 208-289.

[8] B.-W. Schulze, Pseudo-differential boundary value problems, conical singularities, and asymptotics, Akademie Verlag, Berlin, 1994.

[9] B.-W. Schulze, Boundary value problems and singular pseudo-differential operators, J. Wiley, Chichester, 1998.

[10] B.-W. Schulze, Operators with symbol hierarchies and iterated asymptotics, Publications of RIMS, Kyoto University, 38, 4 (2002), 735-802.

Institut für Mathematik, Universität Potsdam, 601553 Potsdam

E-mail address: schulze@math.uni-potsdam.de 